\title{
Safety and efficacy of anti-tau monoclonal antibody gosuranemab in progressive supranuclear palsy: a phase 2, randomized, placebo-
}

\section{controlled trial}

Authors: Tien Dam ${ }^{1 *}$, Adam L. Boxer², Lawrence I. Golbe ${ }^{3}$, Günter U. Höglinger ${ }^{4-6}$, Huw R. Morris $^{7}$, Irene Litvan ${ }^{8}$, Anthony E. Lang ${ }^{10}$, Jean-Christophe Corvol ${ }^{11}$, Ikuko Aiba ${ }^{12}$, Michael Grundman ${ }^{8,9}$, Lili Yang ${ }^{1}$, Beth Tidemann-Miller ${ }^{1}$, Joseph Kupferman ${ }^{1}$, Kristine Harper ${ }^{1}$, Kubra Kamisoglư ${ }^{1}$, Michael J. Wald ${ }^{1}$, Danielle L. Graham ${ }^{1}$, Liz Gedney ${ }^{1}$, John O’Gorman ${ }^{1}$, Samantha Budd Haeberlein"; PASSPORT Study Group*

*A list of the PASSPORT Study Group investigators and their affiliations appears in Supplementary Materials.

${ }^{1}$ Biogen, Cambridge, MA, USA. ${ }^{2}$ Memory and Aging Center, Department of Neurology, University of California, San Francisco, CA, USA. ${ }^{3}$ Rutgers Robert Wood Johnson Medical School, New Brunswick, NJ, USA. ${ }^{4}$ Department of Neurology, Technische Universität München, Munich, Germany. ${ }^{5}$ German Center for Neurodegenerative Diseases (DZNE), Munich, Germany. ${ }^{6}$ Department of Neurology, Hanover Medical School, Hanover, Germany. ${ }^{7}$ National Hospital for Neurology and Neurosurgery, London, UK. ${ }^{8}$ University of California, Parkinson and Other Movement Disorders Center, San Diego, CA, USA. ${ }^{9}$ Global R\&D Partners, LLC, San Diego, CA, USA. ${ }^{10}$ Edmond J. Safra Program in Parkinson's Disease and the Rossy PSP Centre, Toronto Western Hospital and the University of Toronto, Toronto, ON, Canada.

${ }^{11}$ Sorbonne Université, Assistance Publique Hôpitaux de Paris, INSERM, CNRS, Institut du Cerveau - Paris Brain Institute - ICM, Hôpital Pitié-Salpêtrière, Paris, France. ${ }^{12}$ Department of Neurology, National Hospital Organization Higashinagoya National Hospital, Nagoya, Japan. 
${ }^{*}$ Corresponding author: Tien Dam, Biogen, 225 Binney St, Cambridge, MA 02142, USA; email: tien.dam@biogen.com 


\section{ABSTRACT}

A randomized, double-blind, placebo-controlled, 52-week study (NCT03068468) evaluated gosuranemab, an anti-tau monoclonal antibody, for progressive supranuclear palsy (PSP). In total, 486 participants dosed were assigned to gosuranemab $(n=321)$ or placebo $(n=165)$. Efficacy was not demonstrated on adjusted mean change of PSP Rating Scale score at week 52 between gosuranemab and placebo (10.4 versus 10.6 ; $P=0.85$; primary endpoint) or secondary endpoints, resulting in discontinuation of the open-label long-term extension. Unbound N-terminal tau in cerebrospinal fluid decreased by $98 \%$ with gosuranemab and increased by $11 \%$ with placebo $(P<0.0001)$. Incidences of AEs and deaths were similar between groups. This well-powered study suggests $\mathrm{N}$-terminal tau neutralization does not translate to clinical efficacy. 


\section{Introduction}

Progressive supranuclear palsy (PSP) is a rare, aggressive, rapidly progressing, neurodegenerative primary tauopathy characterized by physical and cognitive impairments..$^{1,2}$ In Richardson syndrome, the first-described and most recognized and common form of PSP, ${ }^{1,3}$ patients typically display symptoms of postural instability, falls, slowing of vertical saccades, axial rigidity and neuropsychiatric changes ${ }^{2,4,5}$ beginning at a mean of 67.2 years of age ${ }^{6}$ with additional symptoms, including dysphagia, emerging as the disease progresses. ${ }^{4}$ There are no approved therapies for either the neuroprotective or symptomatic management of PSP, and death occurs within a median of 7.3 years after symptom onset. ${ }^{1,6}$ This compares with a life expectancy of approximately 17.7 years for the US population at age 67 (https://www.ssa.gov/oact/STATS/table4c6.html). Neuropathological examination remains the gold standard for diagnosing $\mathrm{PSP}^{7}$ and reveals abnormal deposits of predominantly fourmicrotubule binding domain repeat (4R) tau protein in the brainstem, deep cerebellar nuclei, basal ganglia and neocortex. ${ }^{8}$ The location of these abnormal tau deposits, which include astrocytic tufts, neurofibrillary tangles and oligodendrocytic coiled bodies, ${ }^{9}$ varies among the PSP phenotype variants and correlates with disease severity and other clinical features of the disease. ${ }^{8}$

Tau is primarily an intracellular protein ${ }^{10}$; however, a variety of tau fragments can be found extracellularly. ${ }^{11,12} \mathrm{~N}$-terminal tau fragments (i.e., tau lacking the microtubule binding region and C-terminus sequences) are especially abundant in cerebrospinal fluid (CSF). ${ }^{12,13}$ Studies in which mice developed tau pathology after being injected with recombinant $4 \mathrm{R}$ tau or specimens from individuals with $4 \mathrm{R}$ tauopathies ${ }^{14-16}$ suggest that abnormal tau, i.e., extracellular $\mathrm{N}$-terminal tau, may drive the spread of tau pathology from neuron to neuron in tauopathies such as PSP and Alzheimer's disease. ${ }^{10}$ 
Gosuranemab (formerly BMS-986168/IPN007/BIIB092) is a humanized immunoglobulin G4P monoclonal antibody directed against the $\mathrm{N}$-terminal tau released by neurons and found extracellularly in the interstitial fluid (ISF) and CSF. Gosuranemab has shown high affinity for fibrillar tau derived from patients with PSP and Alzheimer's disease, and transgenic mice (rTg4510) dosed weekly for 8 weeks with IPN002, a murine version of gosuranemab, had reduced levels of unbound tau in CSF and ISF at 57 days (Golonzhka, O. et al. Functional characterization of anti-tau monoclonal antibody BIIB092. International Conference on Alzheimer's and Parkinson's Diseases. March 26-31, 2019; Lisbon, Portugal). Similarly, cynomolgus monkeys administered a single dose of gosuranemab had reductions in unbound $\mathrm{N}$-terminal tau in ISF (Czerkowicz, J. et al. Pharmacokinetic and target engagement analysis of anti-tau antibody gosuranemab [BIIB092] in cynomolgus monkey central nervous system fluid compartments. Alzheimer's Association International Conference. July 14 - 18, 2019; Los Angeles, CA USA). In a phase 1 study, single doses of gosuranemab in healthy participants reduced unbound CSF $\mathrm{N}$-terminal tau by an average of up to $97 \%$ at day 29 , with doses of $>210$ mg producing persistent unbound N-terminal tau suppression over 12 weeks, with no deaths, serious adverse events (AEs) or discontinuations due to an AE. ${ }^{17}$ In participants with PSP, a randomized, placebo-controlled, multiple ascending dose study revealed that gosuranemab treatment at doses up to $2,100 \mathrm{mg}$ for 12 weeks was well tolerated and reduced CSF unbound $\mathrm{N}$-terminal tau by an average of $90 \%$ at all doses, indicating target engagement. ${ }^{18} \mathrm{We}$ aimed to explore the hypothesis that neutralization of central nervous system $\mathrm{N}$-terminal tau using a targeted N-terminal antibody would be an effective therapy for the primary tauopathy PSP. Here, we report the outcomes of the placebo-controlled 52-week period of the PASSPORT study (NCT03068468), which was conducted to evaluate the efficacy, safety and tolerability of gosuranemab in participants with PSP. 


\section{Results}

Six hundred sixty-two individuals were assessed for eligibility; 490 were randomized and the 486 participants dosed were assigned to receive gosuranemab $(n=321)$ or placebo $(n=$ 165; Extended Data Fig. 1). Demographic characteristics were similar in the two treatment groups (Table 1). The mean (SD) time from onset of symptoms was 3.24 (1.38) years and the mean (SD) time since diagnosis was 1.66 (1.34) years. Overall, the proportion of participants receiving Parkinson's disease medications at baseline was $71 \%$. The mean (SD) baseline score on the 28-item Progressive Supranuclear Palsy Rating Scale (PSPRS) was 36.7 (10.34) and on the Clinical Global Impression of Severity (CGI-S) was 4.0 (0.91); 71.4\% of participants scored $>170$ seconds on the Color Trails Test part 2.

There was no significant difference between the gosuranemab and placebo groups in the primary efficacy endpoint: change from baseline at week 52 in the PSPRS score (Table 2, Fig. 1). Exploratory analyses of the primary efficacy endpoint including adding Color Trails 2 test by visit interaction and adding Color Trails 2 test by visit interaction, Color Trails 2 test by treatment interaction, and Color Trails 2 test by visit by treatment group interaction to the primary mixed model repeated measures model showed similar results (Supplementary Table S1). Because enrollment was low in a few countries (Supplementary Table S2), no exploratory analysis that including country as a fixed effect in the model was performed because this may have resulted in model convergence issues. Similarly, no significant differences were seen between treatment groups in the key secondary efficacy outcomes of adjusted mean change from baseline in the International Parkinson and Movement Disorder Society-sponsored revision of the Unified Parkinson's Disease Rating Scale (MDS-UPDRS) Part II score and PSP cognitive composite z-score, adjusted mean Clinical Global Impression of Change (CGI-C) score, and adjusted mean change in score from baseline in the Progressive Supranuclear Palsy Quality of Life scales (PSP-QoL) (Table 2). No differences between treatment groups in 
absolute change from baseline in all magnetic resonance imaging (MRI) volumetric measures were observed (Table 2).

Serum and CSF gosuranemab concentrations increased after the administration of gosuranemab. Changes in CSF unbound N-terminal tau were significantly different between the gosuranemab and placebo groups, with an adjusted mean decrease of $98.11 \%$ at week 52 for the gosuranemab group and an adjusted mean increase of $10.96 \%$ for the placebo group $(P<$ 0.0001; Table 2; Supplementary Fig S1a). No difference between treatment groups was seen in other exploratory biomarkers, including neurofilament light chain (Table 2; Supplementary Fig S1b).

Safety results are presented in Table 3 . Incidence of AEs, serious AEs, AEs leading to study drug discontinuation and deaths were similar between the placebo and gosuranemab groups. There were 16 (4.9\%) and 8 (4.9\%) deaths in the gosuranemab and placebo groups, respectively, during the placebo-controlled period. The most common causes of death (in at least two participants) were PSP (gosuranemab, $n=4$ ); aspiration pneumonia (placebo, $n=4$; gosuranemab, $n=2$ ), failure to thrive (gosuranemab, $n=2$ ), pneumonia (gosuranemab, $n=2$ ) and respiratory failure (placebo, $n=2$ ); unknown cause of death was reported for two gosuranemab-treated participants. The most common AEs ( $\geq 10 \%$ in either group) were fall, urinary tract infection, skin laceration, contusion, constipation and headache (Table 3). Among AEs that occurred in at least $5 \%$ of participants in either group, those with an incidence at least $2 \%$ higher in gosuranemab- than placebo-treated participants were, respectively, fall $(59.3 \%$ and $53.7 \%)$, skin laceration (13.3\% and $11.1 \%)$, skin abrasion (8.6\% and 6.2\%), musculoskeletal pain (5.9\% and 3.7\%) and pneumonia (5.2\% and 3.1\%) (in gosuranemab and placebo, respectively; Table 4).

While the preferred term of pneumonia was more common in the gosuranemab group relative to placebo, the opposite pattern was observed for aspiration pneumonia (gosuranemab: $1.9 \%$, placebo: $4.9 \%$ ). Post hoc analyses showed that the incidence of pneumonia or aspiration 
pneumonia (gosuranemab: $6.5 \%$, placebo: $8.0 \%$ ) and that of more broadly defined events of infectious pneumonia/lower respiratory infection and aspiration events (gosuranemab: 12.7\%, placebo: $15.4 \%$ ) were similar between the treatment groups (Table 5).

Because falls are common in patients with PSP, not all falls were required to be reported as AEs in this study. Criteria for reporting falls as AEs included falls that were worsening, resulted in injury, were inconsistent with PSP or were associated with a concomitant medication. To explore whether the incidence of falls more generally (rather than only those reported as AEs) increased in the gosuranemab group, efficacy assessments were examined for evidence that falls were different between the treatment groups. A shift analysis of falls (item 5) in the PSPRS at week 52 indicated similar proportions of participants from the gosuranemab and placebo groups had shifts of their scores to worse than baseline $(40.6 \%$ and $43.9 \%$, respectively) and better than baseline (21.9\% and $22.3 \%$, respectively). Similarly, no differences between treatment groups were observed in efficacy data pertaining to balance (PSPRS item 27) or gait (item 26).

There were no hematology or serum chemistry grade 3 or 4 abnormalities that occurred in $2 \%$ or more of gosuranemab-treated participants and were more common than in placebotreated participants.

\section{DISCUSSION}

In this well-powered phase 2 study, 52 weeks of gosuranemab treatment did not demonstrate benefit in patients with possible or probable PSP in the primary efficacy outcome (change from baseline at week 52 in PSPRS) or in any secondary efficacy outcomes, despite increases in serum and CSF gosuranemab concentrations and a mean percentage decrease of $98 \%$ in CSF unbound N-terminal tau after gosuranemab administration. Disease progression as measured over 52 weeks on the PSPRS with both placebo (10.6 points/year) and gosuranemab (10.4 points/year) was similar to that of historical controls (approximately 11 points/year). ${ }^{19}$ The 
overall incidence of AEs was similar in the placebo and gosuranemab groups. The most common AE in this study was fall, which was reported in $59.3 \%$ of gosuranemab and $53.7 \%$ of placebo participants, respectively. Given that falling is a core clinical feature of $\mathrm{PSP}^{2,5}$ not all falls were required to be reported as AEs in this study. Analyses of falls based on PSPRS data, which would be expected to encompass falls more generally (rather than the subset reported as AEs), did not suggest a difference between the treatment groups. Furthermore, similar analyses of PSPRS data on gait and balance did not suggest differences between the groups that could account for a difference in fall risk. The incidence of fatal events was balanced between the placebo and gosuranemab groups, and causes of death were generally consistent with the underlying disease progression of PSP. ${ }^{20}$ The lack of efficacy of gosuranemab for slowing disease progression in PSP could be related to the study's inclusion of patients at a stage of disease where neutralization of cell-to-cell transmission of tau no longer plays a role in disease progression, the inability of gosuranemab to neutralize central (and presumably C-terminal) fragments of tau that may be more strongly linked to toxic gain of function in tauopathies, an insufficient concentration in the brain parenchyma and ISF that may have higher concentrations of extracellular tau than CSF, or the possibility that cell-to-cell transmission of tau species demonstrated in preclinical models is not a major pathogenic component of human disease and, therefore, did not translate to this well-conducted human efficacy study.

Progression of midbrain atrophy was similar between treatment arms and to that reported in previous studies, ${ }^{19,21}$ further indicating a lack of treatment effect at the anatomical level. Midbrain atrophy is a hallmark sign of PSP, which can differentiate PSP from other parkinsonian syndromes, ${ }^{22}$ and midbrain volume loss is the anatomical change most highly correlated with changes in PSPRS score at 52 weeks. ${ }^{23,24}$ Comparable rates of atrophy in other PSP-relevant brain regions were also observed in the two treatment arms. 
On the basis of these negative efficacy findings, the unmet need for an efficacious treatment for PSP remains. Agents that have previously failed to demonstrate therapeutic benefit in large, double-blind trials include davunetide, a peptide hypothesized to promote microtubule stability and reduce tau phosphorylation that was investigated in a phase $2 / 3$ study, ${ }^{19}$ tideglusib, a glycogen synthase kinase 3 inhibitor investigated in a phase 2 study, ${ }^{25}$ riluzole, a glutamatergic signaling modulator ${ }^{26}$ investigated in a phase 3 study, ${ }^{27}$ and ABBV$8 \mathrm{E} 12$, another $\mathrm{N}$-terminal tau antibody investigated in a phase 2 study. ${ }^{28}$

The failure to demonstrate efficacy for gosuranemab in this study was not likely the result of weaknesses in study design, study duration or sample size. The study was well designed to detect any potential benefits of gosuranemab and featured various instruments and assessment tools, including those developed specifically for individuals with PSP (i.e., the PSPRS and PSP-QoL), ${ }^{6,29}$ together with instruments such as the MDS-UPDRS Part II and CGI$\mathrm{C}$, which are used to assess physical disability and cognitive/mental impairment in other populations such as patients with Parkinson's disease, Alzheimer's disease or dementia. ${ }^{30} \mathrm{We}$ also utilized the PSP cognitive composite battery, which was developed during the course of this study to evaluate cognitive deficits in PSP. The study duration and sample size were sufficient to detect a meaningful effect in PSPRS.

Although neuropathology is the gold standard for PSP diagnosis, the study enrollment criteria optimized specificity and sensitivity by utilizing the National Institute of Neurological Disorders and Stroke and Society for PSP possible or probable diagnostic criteria, which have 93-100\% specificity and $50-83 \%$ sensitivity with autopsy-confirmed PSP cases, ${ }^{5,7}$ and by increasing the duration of progressive history of postural instability or falls from 1 to 3 years to improve sensitivity, consistent with the recent MDS diagnostic criteria for PSP. ${ }^{2}$ Participants enrolled in PASSPORT were similar to participants enrolled in other PSP clinical studies with regard to age, sex and baseline disease characteristics, including scores on the PSPRS, MDS- 
UPDRS Part II, CGI-S and Mini-Mental State Examination. ${ }^{19,25}$ However, the National Institute of Neurological Disorders and Stroke and Society for PSP criteria (as well as the MDS criteria) cannot differentiate PSP-Richardson syndrome from PSP-parkinsonism, ${ }^{31}$ which may have biased the results because PSP-Richardson syndrome progresses faster with regard to survival time and frequency of cognitive deficits. ${ }^{32}$

Instead, the lack of efficacy may be due in part to the relatively advanced stage of disease of the PSP study population, although the time since diagnosis was shorter than in the tideglusib study (1.66 years versus 3.2 years). ${ }^{25}$ Baseline scores on various instruments and assessments indicate participants had moderate disease severity and impaired motor activity and cognitive function. ${ }^{6,33,34}$ Therefore, identification of individuals earlier in the disease process may provide a study population more amenable to therapy. However, including patients at an earlier stage remains challenging because of the disease's latency from onset to diagnosis. ${ }^{35}$ There was an approximately 1.5-year average delay from symptom onset to diagnosis of PSP in the PASSPORT study population. Differentiating PSP from similar diseases is challenging, especially early in the course of the disease before pathognomonic signs have developed; an initial diagnosis of Parkinson's disease can lead to a significant delay in the diagnosis of PSP. $4,36,37$

The MDS PSP diagnostic criteria were developed to optimize early, sensitive and specific clinical diagnosis of probable, possible, or suggestive of PSP, ${ }^{2}$ and were shown to have an overall $88 \%$ sensitivity and $86 \%$ specificity in an autopsy-based cohort. ${ }^{38}$ The criteria expanded the clinical spectrum of PSP by incorporating conditions suggestive of PSP and a variety of symptomatic phenotypes, ${ }^{2}$ but the rate of progression of these phenotypes on the PSPRS is unknown. Further work in developing reliable fluid or imaging biomarkers specifically for 4R tauopathies such as PSP, for early and accurate differential diagnosis, will enable clinical trials at initial stages of disease, when therapies are more likely to be efficacious. 
Neuropathological, genetic and preclinical data point to a causative role for tau in PSP, supporting tau as a therapeutic target. ${ }^{1,14,16,39}$ Findings from an earlier study of gosuranemab in participants with PSP were promising; pharmacokinetic and pharmacodynamic data indicated gosuranemab penetrated the central nervous system and bound to and reduced levels of unbound N-terminal tau. ${ }^{18}$ In this study, following monthly administration of gosuranemab $2,000 \mathrm{mg}$, a robust and persistent lowering of CSF unbound $\mathrm{N}$-terminal tau by a mean of $98 \%$ was observed at week 52, demonstrating strong target engagement in the CSF of participants with PSP. Thus, because there are no biomarkers available for identifying PSP patients early in the disease, and these patients have well-established clinical characteristics of PSP, it is possible that the proposed cell-to-cell spread via extracellular $\mathrm{N}$-terminal is well advanced, and intervention with gosuranemab at this stage of disease does not result in clinical benefit.

Alternatively, while preclinical data have supported the hypothesis that tau pathology spreads via extracellular transmittable tau species, ${ }^{10}$ and shown that $\mathrm{N}$-terminal tau bound to gosuranemab is 'neutralized' in primary mouse neurons and subsequent neuronal uptake or spreading is reduced, ${ }^{40}$ these findings did not translate to an effect on slowing disease progression in human PSP patients. Since cell-to-cell spread of extracellular tau species has never been demonstrated in a living human, it remains possible that this phenomenon is not central to the pathology of PSP and other tauopathies, but is a byproduct of the pathophysiology of human tauopathies. In animal models, tau species are predominantly N-terminal and midregion fragments of tau. Extracellular tau can impact the electrophysiological activity of neurons. ${ }^{41-43}$ In animal models, pathologic tau may be released into the extracellular space and internalized by neighboring cells where they can seed intracellular tau aggregation and may spread along neuronal networks to interconnected neurons and adjacent glial cells from one neuroanatomically connected brain region to another and thus propagate tau pathology..$^{10,44,45}$ The hypothesis of transcellular tau spreading is supported by data showing that exogenously 
applied recombinant tau fibrils or tau aggregates present in homogenates prepared from brains of patients with $A D$ can enter cells in culture or in vivo and seed tau pathology. ${ }^{46,47}$

Although CSF tau levels are not increased in PSP patients, preclinical evidence suggests a gradient exists between CSF extracellular tau levels and ISF tau levels. In nonhuman primates and transgenic mice ( $\mathrm{Tg} 4510)$, the mean levels of unbound $\mathrm{N}$-terminal tau are approximately 10-20 fold higher in ISF as compared with ventricular or lumbar CSF (Czerkowicz, J. et al. Pharmacokinetic and target engagement analysis of anti-tau antibody gosuranemab [BIIB092] in cynomolgus monkey central nervous system fluid compartments. Alzheimer's Association International Conference. July 14 - 18, 2019; Los Angeles, CA USA; Czerkowicz, J. et al. Anti-tau antibody BIIB092 binds secreted tau in preclinical models and Alzheimer's Disease cerebrospinal fluid. Alzheimer's Association International Conference. July 22-26, 2018, Chicago, IL USA). It was hypothesized that an increase in extracellular N-terminal tau levels may be present in PSP ISF even though that is not detected in CSF. In addition, studies characterizing BIIB092's ability to attenuate tau spreading (Golonzhka, O. et al. Functional characterization of anti-tau monoclonal antibody BIIB092. International Conference on Alzheimer's and Parkinson's Diseases. March 26-31, 2019; Lisbon, Portugal) suggested that extracellular tau has a role in PSP pathogenesis. For example, immunodepletion studies in clinical samples demonstrated that BIIB092 immunodepletes seeding competent tau from PSP and $A D$ brain homogenates and BIIB092 immunodepleted seeding competent tau present in postmortem PSP CSF samples and resulted in a decrease in tau aggregation.

Therefore, while the N-terminal tau target was engaged by gosuranemab, and led to a neutralization of $\mathrm{N}$-terminal tau, this may not have led to a change in concentration of $\mathrm{N}$-terminal tau fragments. The possibility exists that N-terminal tau bound to gosuranemab, although not reduced, is inactive and is no longer a component of transmissible tau in PSP. As different strains of transmissible tau and varying tau aggregate structures are found in different 
tauopathies, ${ }^{16,48}$ it is possible that the strain of transmissible tau diverges in the preclinical tau transgenic mouse models (in which gosuranemab demonstrated an effect) from those found in patients with PSP, where no effect was seen. Of note, the N-terminal targeted antibody tilavonemab (ABBV-8E12), which targets a similar epitope to gosuranemab, also failed to demonstrate any clinical effects in a recent phase 2 clinical trial in PSP. ${ }^{49}$ The study was terminated early after an interim analysis showed no difference in change in PSPRS with tilavonemab (2000 $\mathrm{mg}$ or $4000 \mathrm{mg}$ ) versus placebo at Week 52 . The authors speculated this may be the result of an inability of tilavonemab to target the pathological tau species responsible for PSP or achievement of only subtherapeutic levels of tilavonemab in the central nervous system. Since patients with PSP have comparable or lower levels of CSF tau (including Nterminal tau) than age-matched controls or individuals with Alzheimer's disease, ${ }^{50-52}$ it is also possible that monoclonal antibodies targeting $\mathrm{N}$-terminal tau epitopes may be less efficacious in PSP than other tauopathies. Alternatively, the presence of tau in nonneuronal cells including astrocytes and oligodendrocytes, in the brains of PSP and other primary tauopathy patients, ${ }^{8,53}$ suggests an as yet unidentified mechanism, beyond or in place of transneuronal spread, could be more relevant in these patients. Therefore, alternative antibodies, antisense oligonucleotides and approaches that target other disease mechanisms, such as neuroinflammation and mitochondrial function, may be future therapies for PSP. ${ }^{54}$

We consider this study to be a high-quality dataset with multiple endpoints across multiple domains, in the largest population of patients with PSP studied to date. The number of patients lost to follow-up and frequency of missing data were lower than projected. Nevertheless, these findings have now revealed that high levels of gosuranemab bound to $\mathrm{N}$ terminal tau in the CSF do not translate to clinical efficacy in patients with PSP with average time from onset of symptoms of $>3$ years. Based on these results, Biogen has discontinued the development of gosuranemab for PSP and other primary tauopathies and has terminated the 
open-label extension of this study. The development of gosuranemab for mild cognitive impairment due to Alzheimer's disease or mild Alzheimer's disease is ongoing in the phase 2 TANGO study (NCT03352557). 


\section{References}

1. Boxer, A. L. et al. Advances in progressive supranuclear palsy: new diagnostic criteria, biomarkers, and therapeutic approaches. Lancet Neurol. 16, 552-563 (2017).

2. Höglinger, G. U. et al; Movement Disorder Society-endorsed PSP Study Group. Clinical diagnosis of progressive supranuclear palsy: the movement disorder society criteria. Mov. Disord. 32, 853-864 (2017).

3. Picillo, M. et al. Motor, cognitive and behavioral differences in MDS PSP phenotypes. J. Neurol. 266, 1727-1735 (2019).

4. Golbe, L. I. Progressive supranuclear palsy. Semin. Neurol. 34, 151-159 (2014).

5. Litvan, I. et al. Clinical research criteria for the diagnosis of progressive supranuclear palsy (Steele-Richardson-Olszewski syndrome): report of the NINDS-SPSP international workshop. Neurology. 47, 1-9 (1996).

6. Golbe, L. I. \& Ohman-Strickland, P. A. A clinical rating scale for progressive supranuclear palsy. Brain. 130, 1552-1565 (2007).

7. Respondek, G. et al. Accuracy of the National Institute for Neurological Disorders and Stroke/Society for Progressive Supranuclear Palsy and neuroprotection and natural history in Parkinson plus syndromes criteria for the diagnosis of progressive supranuclear palsy. Mov. Disord. 28, 504-509 (2013).

8. Dickson, D. W., Ahmed, Z., Algom, A. A., Tsuboi, Y. \& Josephs, K. A. Neuropathology of variants of progressive supranuclear palsy. Curr. Opin. Neurol. 23, 394-400 (2010).

9. Dickson, D. W. Parkinson's disease and parkinsonism: neuropathology. Cold Spring Harb. Perspect. Med. 2, a009258 (2012). 
10. Fuster-Matanzo, A., Hernández, F. \& Ávila, J. Tau spreading mechanisms; implications for dysfunctional tauopathies. Int. J. Mol. Sci. 19, 645 (2018).

11. Meredith, J. E., Jr. et al. Characterization of novel CSF Tau and ptau biomarkers for Alzheimer's disease. PLoS One. 8, e76523 (2013).

12. Bright, J. et al. Human secreted tau increases amyloid-beta production. Neurobiol. Aging. 36, 693-709 (2015).

13. Sato, C. et al. Tau kinetics in neurons and the human central nervous system. Neuron. $\mathbf{9 7}$, 1284-1298 (2018).

14. Clavaguera, F. et al. Brain homogenates from human tauopathies induce tau inclusions in mouse brain. Proc. Natl. Acad. Sci. U. S. A. 110, 9535-9540 (2013).

15. Mocanu, M. M. et al. The potential for beta-structure in the repeat domain of tau protein determines aggregation, synaptic decay, neuronal loss, and coassembly with endogenous Tau in inducible mouse models of tauopathy. J. Neurosci. 28, 737-748 (2008).

16. Sanders, D. W. et al. Distinct tau prion strains propagate in cells and mice and define different tauopathies. Neuron. 82, 1271-1288 (2014).

17. Qureshi, I. A. et al. A randomized, single ascending dose study of intravenous BlIB092 in healthy participants. Alzheimers Dement. (N. Y.). 4, 746-755 (2018).

18. Boxer, A. L. et al. Safety of the tau-directed monoclonal antibody BIIB092 in progressive supranuclear palsy: a randomised, placebo-controlled, multiple ascending dose phase $1 \mathrm{~b}$ trial. Lancet Neurol. 18, 549-558 (2019).

19. Boxer, A. L. et al. Davunetide in patients with progressive supranuclear palsy: a randomised, double-blind, placebo-controlled phase 2/3 trial. Lancet Neurol. 13, 676-685 (2014). 
20. Papapetropoulos, S., Singer, C., McCorquodale, D., Gonzalez, J. \& Mash, D. C. Cause, seasonality of death and co-morbidities in progressive supranuclear palsy (PSP). Parkinsonism Relat. Disord. 11, 459-463 (2005).

21. Höglinger, G. U. et al. Longitudinal magnetic resonance imaging in progressive supranuclear palsy: A new combined score for clinical trials. Mov. Disord. 32, 842-852 (2017).

22. Whitwell, J. L. et al; Movement Disorder Society-endorsed PSP Study Group. Radiological biomarkers for diagnosis in PSP: where are we and where do we need to be? Mov. Disord. 32, 955-971 (2017).

23. Josephs, K. A. et al. Modeling trajectories of regional volume loss in progressive supranuclear palsy. Mov. Disord. 28, 1117-1124 (2013).

24. Tsai, R. M. et al; AL-108-231 Investigators. Clinical correlates of longitudinal brain atrophy in progressive supranuclear palsy. Parkinsonism Relat. Disord. 28, 29-35 (2016).

25. Tolosa, E. et al; TAUROS Investigators. A phase 2 trial of the GSK-3 inhibitor tideglusib in progressive supranuclear palsy. Mov. Disord. 29, 470-478 (2014).

26. Doble, A. The pharmacology and mechanism of action of riluzole. Neurology. 47, S233241 (1996).

27. Bensimon, G. et al; NNIPPS Study Group. Riluzole treatment, survival and diagnostic criteria in Parkinson plus disorders: the NNIPPS study. Brain. 132, 156-171 (2009).

28. Vaswani, P. A. \& Olsen, A. L. Immunotherapy in progressive supranuclear palsy. Curr. Opin. Neurol. 33, 527-533 (2020).

29. Schrag, A. et al. Measuring quality of life in PSP: the PSP-QoL. Neurology. 67, 39-44 (2006). 
30. Goetz, C. G. et al. Movement Disorder Society-sponsored revision of the Unified Parkinson's Disease Rating Scale (MDS-UPDRS): scale presentation and clinimetric testing results. Mov. Disord. 23, 2129-2170 (2008).

31. Shoeibi, A. et al. Are the International Parkinson disease and Movement Disorder Society progressive supranuclear palsy (IPMDS-PSP) diagnostic criteria accurate enough to differentiate common PSP phenotypes? Parkinsonism Relat. Disord. 69, 34-39 (2019).

32. Respondek, G. et al; Movement Disorder Society-endorsed PSP Study Group. The phenotypic spectrum of progressive supranuclear palsy: a retrospective multicenter study of 100 definite cases. Mov. Disord. 29, 1758-1766 (2014).

33. Martínez-Martín, P. et al. Parkinson's disease severity levels and MDS-Unified Parkinson's Disease Rating Scale. Parkinsonism Relat. Disord. 21, 50-54 (2015).

34. Busner, J. \& Targum, S. D. The clinical global impressions scale: applying a research tool in clinical practice. Psychiatry (Edgmont (Pa. : Township)). 4, 28-37 (2007).

35. Mamarabadi, M., Razjouyan, H. \& Golbe, L. I. Is the latency from progressive supranuclear palsy onset to diagnosis improving? Mov. Disord. Clin. Pract. 5, 603-606 (2018).

36. Armstrong, R. A. Visual signs and symptoms of progressive supranuclear palsy. Clin. Exp. Optom. 94, 150-160 (2011).

37. Respondek, G. et al; Movement Disorder Society-Endorsed PSP Study Group. Which ante mortem clinical features predict progressive supranuclear palsy pathology? Mov. Disord. 32, 995-1005 (2017).

38. Ali, F. et al. Sensitivity and specificity of diagnostic criteria for progressive supranuclear palsy. Mov. Disord. 34, 1144-1153 (2019). 
39. Höglinger, G. U. et al; PSP Genetics Study Group. Identification of common variants influencing risk of the tauopathy progressive supranuclear palsy. Nat. Genet. 43, 699-705 (2011).

40. Sopko, R. et al. Characterization of tau binding by gosuranemab. Neurobiol. Dis. 146, 105120 (2020).

41. Bright, J. et al. Human secreted tau increases amyloid-beta production. Neurobiol. Aging. 36, 693-709 (2015).

42. Gómez-Ramos, A., Díaz-Hernández, M., Cuadros, R., Hernández, F. \& Avila, J. Extracellular tau is toxic to neuronal cells. FEBS Lett. 580, 4842-4850 (2006).

43. Gómez-Ramos, A., Díaz-Hernández, M., Rubio, A., Miras-Portugal, M. T. \& Avila, J. Extracellular tau promotes intracellular calcium increase through M1 and M3 muscarinic receptors in neuronal cells. Mol. Cell. Neurosci. 37, 673-681 (2008).

44. Gibbons, G. S., Lee, V. M. Y. \& Trojanowski, J. Q. Mechanisms of Cell-to-Cell Transmission of Pathological Tau: A Review. JAMA neurology. 76, 101-108 (2019).

45. Yamada, K. Extracellular Tau and Its Potential Role in the Propagation of Tau Pathology. Front. Neurosci. 11, 667 (2017).

46. Courade, J. P. et al. Epitope determines efficacy of therapeutic anti-Tau antibodies in a functional assay with human Alzheimer Tau. Acta Neuropathol. 136, 729-745 (2018).

47. Guo, J. L. et al. Unique pathological tau conformers from Alzheimer's brains transmit tau pathology in nontransgenic mice. J. Exp. Med. 213, 2635-2654 (2016).

48. Scheres, S. H., Zhang, W., Falcon, B. \& Goedert, M. Cryo-EM structures of tau filaments. Curr. Opin. Struct. Biol. 64, 17-25 (2020). 
49. Höglinger, G. U. et al. Safety and efficacy of tilavonemab in progressive supranuclear palsy: a phase 2, randomised, placebo-controlled trial. Lancet Neurol. 20, 182-192 (2021).

50. Hall, S. et al. Cerebrospinal fluid concentrations of inflammatory markers in Parkinson's disease and atypical parkinsonian disorders. Sci. Rep. 8, 13276 (2018).

51. Hall, S. et al. Accuracy of a panel of 5 cerebrospinal fluid biomarkers in the differential diagnosis of patients with dementia and/or parkinsonian disorders. Arch. Neurol. 69, 1445-1452 (2012).

52. Wagshal, D. et al. Divergent CSF т alterations in two common tauopathies: Alzheimer's disease and progressive supranuclear palsy. J. Neurol. Neurosurg. Psychiatry. 86, $244-$ 250 (2015).

53. Kovacs, G. G. Invited review: Neuropathology of tauopathies: principles and practice. Neuropathol. Appl. Neurobiol. 41, 3-23 (2015).

54. Shoeibi, A., Olfati, N. \& Litvan, I. Preclinical, phase I, and phase II investigational clinical trials for treatment of progressive supranuclear palsy. Expert Opin. Investig. Drugs. 27, 349-361 (2018).

55. Grimm, M. J. et al; Movement Disorder Society-Endorsed PSP Study Group. Clinical conditions "suggestive of progressive supranuclear palsy"—diagnostic performance. Mov. Disord., doi: 10.1002/mds.28263 (2020).

56. D’Elia, L. F., Satz, P., Uchiyama, C. L. \& White, T. Color Trails Test. Professional Manual (Psychological Assessment Resources, Odessa, FL, 1996).

57. Wolz, R., Aljabar, P., Hajnal, J. V., Hammers, A. \& Rueckert, D.; Alzheimer's Disease Neuroimaging Initiative. LEAP: learning embeddings for atlas propagation. Neuroimage. 49, 1316-1325 (2010). 
58. Guy, W. ECDEU Assessment Manual for Psychopharmacology (U.S. Department of Health, Education, and Welfare, Rockville, MD, 1976).

59. Karantzoulis, S., Novitski, J., Gold, M. \& Randolph, C. The Repeatable Battery for the Assessment of Neuropsychological Status (RBANS): utility in detection and characterization of mild cognitive impairment due to Alzheimer's disease. Arch. Clin. Neuropsychol. 28, 837-844 (2013).

60. Dal Bello-Haas, V., Klassen, L., Sheppard, M. S. \& Metcalfe, A. Psychometric properties of activity, self-efficacy, and quality-of-life measures in individuals with Parkinson disease. Physiother. Can. 63, 47-57 (2011).

61. Miller, E. Verbal fluency as a function of a measure of verbal intelligence and in relation to different types of cerebral pathology. Br. J. Clin. Psychol. 23, 53-57 (1984).

62. Stamelou, M. et al; AL-108-231 Investigators, Tauros Investigators \& MDS-Endorsed PSP Study Group. Power calculations and placebo effect for future clinical trials in progressive supranuclear palsy. Mov. Disord. 31, 742-747 (2016). 


\section{Acknowledgements}

Biogen (Cambridge, MA, USA) sponsored this study and provided medical writing and editorial support for the development of this publication. Suzanne Douthwaite and Meryl Mandle (Excel Medical Affairs, Fairfield, CT, USA) wrote the first draft of the manuscript based on input from the authors, and Jackie Parker (Excel Medical Affairs, Fairfield, CT, USA) copyedited and styled the manuscript per journal requirements. Biogen reviewed and provided feedback on the paper to the authors. All authors had full access to the study data, contributed to data interpretation and manuscript development, provided final approval for submission, and take responsibility for data completeness and accuracy. The corresponding author had final responsibility for the decision to submit for publication.

The authors wish to thank the patients with PSP who participated in the PASSPORT trial, their families, the PASSPORT study group investigators and the clinical research centers where the study occurred. This research was carried out in part by the National Institute for Health Research (NIHR) UCLH Clinical Research Facility in the United Kingdom. The authors also acknowledge Tina Olsson and Miranda Tighe for their contributions to the study design, and Vineeth Kurnala (lead statistical programmer for study) for his contribution to the data analysis.

\section{Authors contributions}

T.D.: data analysis, interpretation and writing of the manuscript; A.L.B.: study design, data collection, data analysis, data interpretation, participant recruitment and writing of the report;

L.I.G.: development and implementation of the primary endpoint instrument, study design, participant recruitment, data collection, data interpretation, writing of the report and approval of the final version of the manuscript; G.U.H.: study design, participant recruitment, data collection, data interpretation, writing of the report and approval of the final version of the manuscript; H.R.M.: study design, data collection, data interpretation, participant recruitment and critical 
review of the report; I.L.: study design, data collection, data interpretation, participant recruitment and critical review of the report; A.E.L.: study design, data analysis, data interpretation, reviewing the manuscript and approval of the final version of the manuscript; J.C.C.: study design, data collection, data interpretation, participant recruitment and reviewing the manuscript; I.A.: study design, participant recruitment, data collection, data interpretation and reviewing the manuscript; M.G.: study design, statistical analysis plan design, data collection, data interpretation and writing of the report; L.Y.: data analysis, data interpretation and writing of the manuscript; B.T.-M.: data analysis, data interpretation and writing of the manuscript; J.K.: data analysis, data interpretation and writing of the report; K.H.: data analysis, data interpretation and writing of the report; K.K.: data analysis, data interpretation and writing of the manuscript; M.J.W.: data collection, data analysis, data interpretation and writing of the report; D.L.G.: study design, data analysis, data interpretation and writing of the manuscript; L.G.: clinical operations and reviewing the manuscript; J.O.: data analysis, data interpretation and writing of the manuscript; S.B.H.: data analysis, interpretation and writing of the manuscript.

\section{Competing interests}

A.L.B. reports consultancy for AGTC, Alector, Arkuda, Arvinas, Asceneuron, AZTherapies, Bioage, GSK, Humana, Lundbeck, Ono, Roche, Samumed, Sangamo, Stealth Therapeutics, Third Rock, Transposon, UCB and Wave and research support from the Association for Frontotemporal Degeneration, Biogen, Bluefield Project to Cure Frontotemporal Dementia, Eli Lilly, Eisai, National Institutes of Health (grant numbers U19AG063911,U54NS092089, R01AG031278), and Tau Research Consortium.

L.I.G. reports consultancy for AbbVie, AlzProtect, Asceneuron, Biogen, CurePSP, Martin, Mitochon, Mitsubishi Tanabe, Pinteon, Retrotope, Roche, Stealth BioTherapeutics and UCB and research support from AbbVie, American Parkinson's Disease Association and Biogen. 
G.U.H. reports consultancy for AbbVie, AlzProtect, Asceneuron, Biogen, Biohaven, Lundbeck, Novartis, Roche, Sanofi and UCB; honoraria for scientific presentations from AbbVie, Bayer Vital, Bial, Biogen, Bristol-Myers Squibb, Roche, Teva, UCB and Zambon; research collaborations with Orion, Prothena and Roche; research support from GE Health, Neuropore, German Research Foundation (DFG; EXC 2145 SyNergy - ID 390857198; HO2402/6-2; HO2402/18-1), the German Federal Ministry of Education and Research (BMBF, 01KU1403A; 01EK1605A), the NOMIS foundation (FTLD project), the EU/EFPIA/Innovative Medicines Initiative [2] Joint Undertaking (IMPRIND grant $n^{\circ} 116060$ ), and VolkswagenStiftung / Lower Saxony Ministry for Science / Petermax-Müller Foundation (Niedersächsisches Vorab).

H.R.M. reports consultancy for AbbVie, Biogen, Biohaven, Denali and UCB; employment by North Thames National Institute for Health Research and University College London; lecture fees/honoraria from Biogen, C4X Discovery, Movement Disorders Society, UCB and Wellcome Trust; research grants from CBD Solutions, Cure Parkinson's Trust, Drake Foundation, Medical Research Council, MND Association, Parkinson's UK and PSP Association; and being a coapplicant on a patent application related to C9ORF72 (method for diagnosing a neurodegenerative disease [PCT/GB2012/052140]).

I.L. reports scientific advisory board membership for Corticobasal Degeneration Solutions and Lundbeck; research support from AbbVie, Biogen, Biohaven, EIP-Pharma, Roche, Lewy Body Association, Michael J. Fox Foundation, National Institutes of Health (grant numbers 5P50AG005131-33, 2R01AG038791-06A, U01NS090259, U01NS100610, U01NS80818, R25NS098999, P20GM109025; U19 AG063911-1; 1R21NS11476401A1), Parkinson Foundation and Parkinson Study Group; employment at University of California San Diego and Chief Editor of Frontiers in Neurology.

A.E.L. reports advisory board membership for AbbVie, AFFiRis, Biogen, Corticobasal Degeneration Solutions, Janssen, Jazz Pharma, Lilly, Lundbeck, Merck, Paladin, 
PhotoPharmics, Roche, Sunovion, Sun Pharma and Theravance; honoraria from AbbVie, Sunovion and Sun Pharma; grants from Brain Canada, Canadian Institutes of Health Research, Corticobasal Degeneration Solutions, Edmond J. Safra Philanthropic Foundation, Michael J. Fox Foundation, Ontario Brain Institute, the Parkinson Foundation and Parkinson Society Canada.

J.-C.C. reports advisory board membership for Pfizer; consultancy for Air Liquide, AlzProtect, Biogen, Bristol-Myers Squibb, Denali and Theranexus; speaker fees from Biogen and Ever Pharma; research grants from Actelion and Michael J. Fox Foundation; and travel grants from MDS.

I.A. reports consultancy for AbbVie and Biogen.

M.G. reports consultancy for Biogen and Bristol-Myers Squibb.

T.D., L.Y., B.T.-M., K.K., M.J.W., D.L.G., L.G., J.O., S.B.H. are employees of and hold stock in Biogen.

J.K. is a former employee of and holds stock in Biogen.

K.H. reports being an independent physician pharmacovigilance professional at Biogen for the PASSPORT study. 


\section{FIGURE LEGEND}

Fig. 1. Change from baseline in total PSPRS over 52 weeks (ITT population). A greater positive change indicates worsening of PSP. Adjusted mean \pm SE (standard error) for each treatment group and $P$ value at Week 52 (placebo, $n=139$; gosuranemab, $n=278$ ) was based on a mixed model for repeated measures change from baseline in PSPRS total score as dependent variable and with fixed effects of treatment group, time (categorical), treatment group by time interaction, baseline PSPRS, baseline PSPRS by time interaction, baseline Color Trails 2 test ( $\leq 170$ or $>170$ seconds) and region. According to the pre-specified hierarchical testing procedure, the primary endpoint was tested two-sided with an alpha level of 0.05 . The baseline (post-baseline) sample sizes reflect the number of participants with a baseline and at least one post-baseline value (number of participants with a baseline and at least one post-baseline value at the visit of interest), respectively.

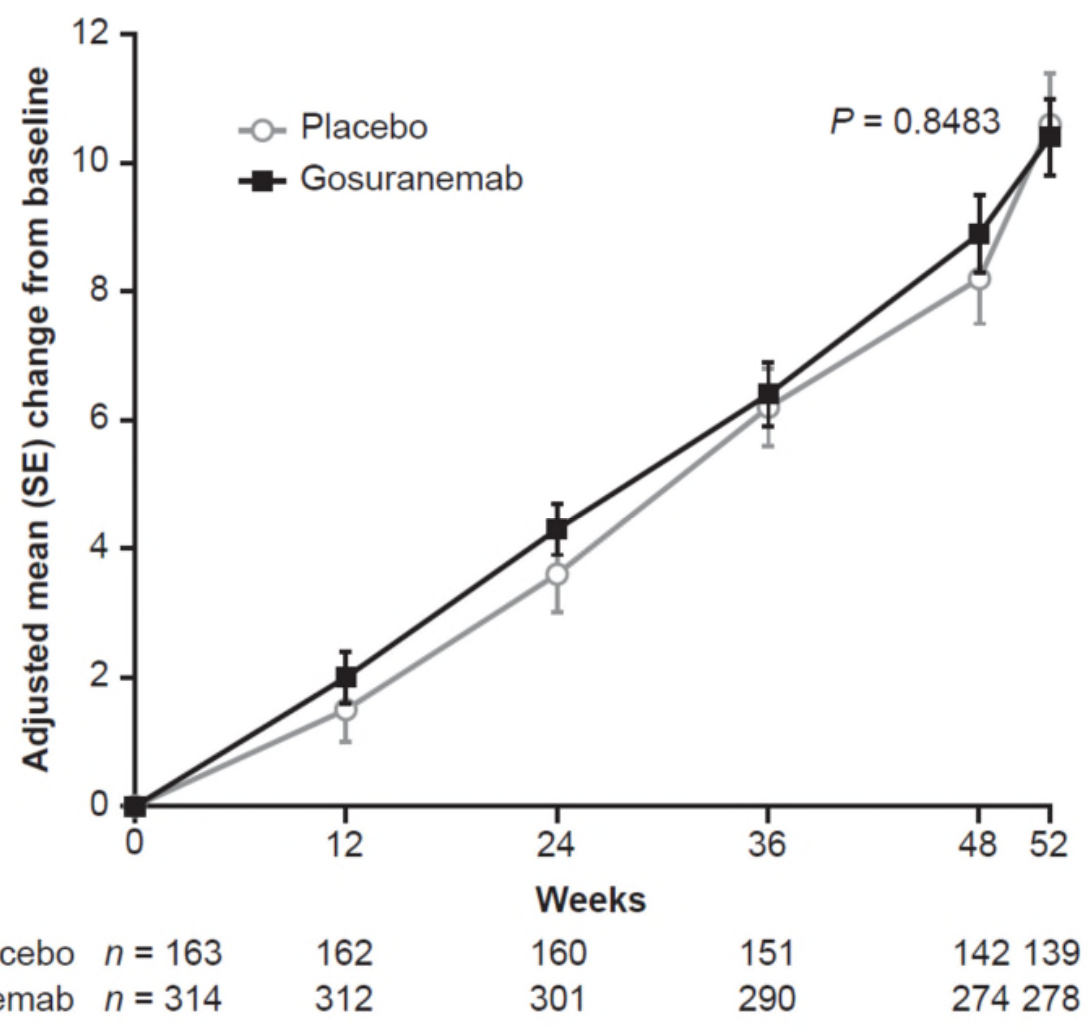


Table 1 Patient demographics and baseline disease characteristics (intention-to-treat population: placebo-controlled period)

\begin{tabular}{|c|c|c|c|}
\hline Characteristic & $\begin{array}{l}\text { Placebo } \\
n=165\end{array}$ & $\begin{array}{c}\text { Gosuranemab } \\
n=321\end{array}$ & $\begin{array}{c}\text { Total } \\
N=486\end{array}$ \\
\hline Age, years & $68.9(6.57)$ & $68.7(7.02)$ & $68.7(6.86)$ \\
\hline Sex, women & $74(44.8)$ & $136(42.4)$ & $210(43.2)$ \\
\hline Race, white & $138(83.6)$ & $281(87.5)$ & $419(86.2)$ \\
\hline $\begin{array}{l}\text { Weight, kg } \\
\text { Time since onset of symptoms, years }\end{array}$ & $\begin{array}{l}n=165 \\
75.3(16.35) \\
3.39(1.38)\end{array}$ & $\begin{array}{c}n=318 \\
75.1(16.14) \\
3.16(1.38)\end{array}$ & $\begin{array}{c}n=483 \\
75.2(16.20) \\
3.24(1.38)\end{array}$ \\
\hline Time since diagnosis, years & $1.80(1.41)$ & $1.59(1.30)$ & $1.66(1.34)$ \\
\hline $\begin{array}{l}\text { Tau haplotype } \\
\text { H1/H1 } \\
\mathrm{H} 1 / \mathrm{H} 2\end{array}$ & $\begin{array}{c}145(87.9) \\
5(3.0)\end{array}$ & $\begin{array}{c}271(84.4) \\
12(3.7)\end{array}$ & $\begin{array}{c}416(85.6) \\
17(3.5)\end{array}$ \\
\hline Concomitant antiparkinsonian medications & $123(74.5)$ & $220(68.5)$ & $343(70.6)$ \\
\hline Dopa and dopa derivatives & $109(66.1)$ & $196(61.1)$ & $305(62.8)$ \\
\hline Adamantane derivatives & $31(18.8)$ & $58(18.1)$ & $89(18.3)$ \\
\hline Total PSPRS score & $37.3(9.93)$ & $36.4(10.54)$ & $36.7(10.34)$ \\
\hline $\begin{array}{l}\text { MDS-UPDRS Part II score } \\
\text { PSP cognitive composite battery, z-score }\end{array}$ & $\begin{array}{c}n=165 \\
23.0(8.76) \\
n=164 \\
0.02(0.66)\end{array}$ & $\begin{array}{c}n=317 \\
22.0(9.28) \\
n=319 \\
-0.03(0.65)\end{array}$ & $\begin{array}{c}n=482 \\
22.4(9.10) \\
n=483 \\
-0.01(0.65)\end{array}$ \\
\hline PSP-QoL & $n=165$ & $n=318$ & $n=483$ \\
\hline Physical score & $39.8(18.24)$ & $40.0(20.15)$ & $39.9(19.50)$ \\
\hline Mental score & $24.4(17.22)$ & $24.5(18.31)$ & $24.5(17.92)$ \\
\hline VAS score & $56.7(25.10)$ & $56.7(22.38)$ & $56.7(23.32)$ \\
\hline $\begin{array}{l}\text { SEADL score } \\
\text { CGI-S score }\end{array}$ & $\begin{array}{c}n=164 \\
56.8(20.4) \\
n=164 \\
4.1(0.86)\end{array}$ & $\begin{array}{c}n=319 \\
57.0(21.7) \\
n=320 \\
4.0(0.94)\end{array}$ & $\begin{array}{c}n=483 \\
56.9(21.3) \\
n=484 \\
4.0(0.91)\end{array}$ \\
\hline Color Trails 2 test $\leq 170$ seconds & $49(29.7)$ & $90(28.0)$ & $139(28.6)$ \\
\hline Phonemic fluency test, words per minute & $12.3(7.54)$ & $10.3(5.89)$ & $11.0(6.56)$ \\
\hline Letter-number sequencing test score & $14.2(5.55)$ & $13.8(5.37)$ & $13.9(5.43)$ \\
\hline Mini-Mental State Examination score & $\begin{array}{c}n=164 \\
26.5(2.68)\end{array}$ & $\begin{array}{c}n=319 \\
26.3(2.69)\end{array}$ & $\begin{array}{c}n=483 \\
26.4(2.69)\end{array}$ \\
\hline \multicolumn{4}{|l|}{ Volume on $\mathrm{MRI}^{\mathrm{a}}, \mathrm{cm}^{3}$} \\
\hline Lateral ventricles & $\begin{array}{c}n=153 \\
43.48(21.893)\end{array}$ & $\begin{array}{c}n=299 \\
41.14(19.929)\end{array}$ & \\
\hline Whole brain & $\begin{array}{c}n=158 \\
1,066.09(103.556)\end{array}$ & $\begin{array}{c}n=310 \\
1,073.89(113.203)\end{array}$ & \\
\hline Midbrain & $\begin{array}{c}n=162 \\
6.60(1.013)\end{array}$ & $\begin{array}{c}n=314 \\
6.69(1.057)\end{array}$ & \\
\hline Pons & $\begin{array}{c}n=146 \\
13.46(2.306)\end{array}$ & $\begin{array}{c}n=296 \\
13.61(2.197)\end{array}$ & \\
\hline CSF unbound N-terminal tau, $\mathrm{pg} / \mathrm{ml}^{\mathrm{b}}$ & $\begin{array}{c}n=37 \\
100.71(62.816)\end{array}$ & $\begin{array}{c}n=85 \\
103.51(125.383)\end{array}$ & \\
\hline CSF neurofilament light chain $\mathrm{pg} / \mathrm{ml}^{\mathrm{b}}$ & $\begin{array}{c}n=37 \\
3156.38(2345.548)\end{array}$ & $\begin{array}{c}n=84 \\
2646.44(1473.790)\end{array}$ & \\
\hline
\end{tabular}

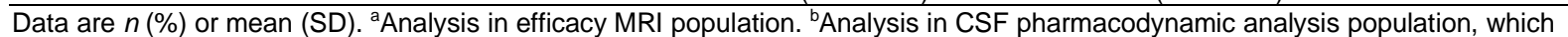
consisted of those participants in the safety population who had at least one sample evaluable for exploratory CSF biomarkers. VAS, visual analog scale. 
Table 2 Change in outcome measures from baseline to week 52 (intention-to-treat population: placebo-controlled period)

\begin{tabular}{|c|c|c|c|c|}
\hline \multirow[t]{2}{*}{ Endpoint } & \multicolumn{2}{|c|}{ Adjusted mean (SE) change at week 52} & \multirow{2}{*}{$\begin{array}{c}\text { Adjusted mean } \\
\text { difference }(95 \% \mathrm{Cl})\end{array}$} & \multirow[t]{2}{*}{$P$ value } \\
\hline & $\begin{array}{l}\text { Placebo } \\
n=165\end{array}$ & $\begin{array}{c}\text { Gosuranemab } \\
\quad n=321\end{array}$ & & \\
\hline Primary endpoint $^{\mathrm{a}}$ & $n=139$ & $n=278$ & & \\
\hline Total PSPRS score & $10.6(0.8)$ & $10.4(0.6)$ & $-0.2(-2.0,1.6)$ & 0.8483 \\
\hline \multicolumn{5}{|l|}{ Key secondary endpoints ${ }^{a}$} \\
\hline MDS-UPDRS Part II score & $\begin{array}{c}n=143 \\
6.7(0.6)\end{array}$ & $\begin{array}{l}n=270 \\
7.0(0.4)\end{array}$ & $0.4(-1.0,1.7)$ & 0.6031 \\
\hline CGI-C score ${ }^{b}$ & $\begin{array}{l}n=138 \\
5.3(0.1)\end{array}$ & $\begin{array}{l}n=271 \\
5.2(0.1)\end{array}$ & $-0.0(-0.2,0.1)$ & 0.7743 \\
\hline $\begin{array}{l}\text { PSP cognitive composite } \\
\text { battery z-score }\end{array}$ & $\begin{array}{c}n=134 \\
-0.283(0.032)\end{array}$ & $\begin{array}{c}n=249 \\
-0.245(0.024)\end{array}$ & $\begin{array}{c}0.038 \\
(-0.036,0.112)\end{array}$ & 0.3180 \\
\hline PSP-QoL & & & & \\
\hline Physical score & $\begin{array}{c}n=142 \\
11.3(1.5)\end{array}$ & $\begin{array}{c}n=264 \\
11.2(1.1)\end{array}$ & $-0.2(-3.6,3.3)$ & 0.9304 \\
\hline Mental score & $\begin{array}{l}n=140 \\
5.6(1.4)\end{array}$ & $\begin{array}{l}n=264 \\
6.1(1.0)\end{array}$ & $0.5(-2.8,3.7)$ & 0.7859 \\
\hline VAS score & $\begin{array}{c}n=141 \\
-3.7(1.8)\end{array}$ & $\begin{array}{c}n=264 \\
-5.4(1.3)\end{array}$ & $-1.7(-5.8,2.5)$ & 0.4297 \\
\hline \multicolumn{5}{|l|}{ Other secondary endpoints } \\
\hline SEADL score ${ }^{a, c}$ & $\begin{array}{c}n=140 \\
-13.7(1.4)\end{array}$ & $\begin{array}{c}n=277 \\
-11.7(1.0)\end{array}$ & $2.0(-1.1,5.2)$ & 0.2084 \\
\hline CGI-S score ${ }^{a}$ & $\begin{array}{l}n=140 \\
0.6(0.1)\end{array}$ & $\begin{array}{l}n=269 \\
0.6(0.0)\end{array}$ & $-0.0(-0.2,0.1)$ & 0.5701 \\
\hline $\begin{array}{l}\text { Phonemic fluency test, words } \\
\text { per minute }\end{array}$ & $\begin{array}{l}n=141 \\
-0.9(0.4)\end{array}$ & $\begin{array}{l}n=273 \\
0.0(0.3)\end{array}$ & $0.9(-0.0,1.8)$ & 0.0517 \\
\hline $\begin{array}{l}\text { Letter-number sequencing test } \\
\text { score }^{\mathrm{a}, \mathrm{c}}\end{array}$ & $\begin{array}{l}n=139 \\
-1.9(0.4)\end{array}$ & $\begin{array}{c}n=271 \\
-1.1(0.3)\end{array}$ & $0.9(0.0,1.7)$ & 0.0387 \\
\hline \multicolumn{5}{|l|}{$\begin{array}{l}\text { MRI, absolute volume change }{ }^{\mathrm{a}, \mathrm{d}} \text {, } \\
\mathrm{cm}^{3}\end{array}$} \\
\hline Lateral ventricles & $\begin{array}{c}n=103 \\
3.823(0.302)\end{array}$ & $\begin{array}{c}n=222 \\
3.802(0.216)\end{array}$ & $\begin{array}{c}-0.021 \\
(-0.726,0.684)\end{array}$ & 0.9527 \\
\hline Whole brain & $\begin{array}{c}n=101 \\
-18.612(1.296)\end{array}$ & $\begin{array}{c}n=210 \\
-19.126(0.950)\end{array}$ & $\begin{array}{c}-0.514 \\
(-3.506,2.478)\end{array}$ & 0.7357 \\
\hline Midbrain & $\begin{array}{c}n=108 \\
-0.116(0.008)\end{array}$ & $\begin{array}{c}n=224 \\
-0.120(0.006)\end{array}$ & $\begin{array}{c}-0.004 \\
(-0.023,0.014)\end{array}$ & 0.6439 \\
\hline Pons & $\begin{array}{c}n=100 \\
-0.198(0.017)\end{array}$ & $\begin{array}{c}n=223 \\
-0.198(0.012)\end{array}$ & $\begin{array}{c}0.000 \\
(-0.039,0.040)\end{array}$ & 0.9864 \\
\hline CSF unbound $\mathrm{N}$-terminal taue,f & $\begin{array}{c}n=24 \\
10.96(4.05)\end{array}$ & $\begin{array}{c}n=61 \\
-98.11(2.60)\end{array}$ & $\begin{array}{c}-109.07 \\
(-118.21,-99.93)\end{array}$ & $<0.0001$ \\
\hline CSF neurofilament light chaine,f & $\begin{array}{c}n=24 \\
8.94(10.066)\end{array}$ & $\begin{array}{c}n=59 \\
14.61(6.513)\end{array}$ & $\begin{array}{c}5.68 \\
(-17.665,29.020)\end{array}$ & 0.6296 \\
\hline
\end{tabular}

ata were analyzed using a mixed model for repeated measures, with change from baseline score as dependent variable and with fixed effects of treatment group, time (categorical), treatment group by time interaction, baseline value, baseline value by time interaction, baseline Color Trails 2 test ( $\leq 170$ or $>170$ seconds) and region. ${ }^{b}$ Adjusted mean (SE) at week 52 . Data were analyzed using a mixed model for repeated measures, with CGI-C as dependent variable and with fixed effects of treatment group, time (categorical), treatment group by time interaction, baseline CGI-S, baseline CGI-S by time interaction, baseline Color Trails 2 test ( $\leq 170$ or $>170$ seconds) and region. ${ }^{\circ}$ Change at week $48 .{ }^{d}$ Analysis in efficacy MRI population. ${ }^{e}$ Analysis in CSF pharmacodynamic analysis population, which consisted of those participants in the safety population who had at least one sample evaluable for exploratory CSF biomarkers. ${ }^{\dagger}$ Adjusted mean (SE) percentage change at week 52 . Data were based on an analysis of covariance model fitted with percentage change from baseline, adjusting for treatment group, baseline value, Color Trails 2 test ( $\leq 170$ or $>170$ seconds) and region. VAS, visual analog scale. Primary and key secondary endpoints were tested according to the pre-specified hierarchical testing procedure. Since the primary endpoint was not significant $(P>0.05)$, the testing was stopped after the primary endpoint. $P$-values reported for key secondary endpoints and all other endpoints were nominal. All $P$-values were tested two-sided. F statistics were used for the statistical testing with the Kenward-Rodgers method used for denominator degrees of freedom in the mixed model for repeated measures analyses. 
Table 3 Safety (safety population)

\begin{tabular}{|c|c|c|}
\hline & $\begin{array}{l}\text { Placebo } \\
n=162\end{array}$ & $\begin{array}{c}\text { Gosuranemab } \\
n=324^{\mathrm{a}}\end{array}$ \\
\hline \multicolumn{3}{|l|}{ Primary safety endpoints } \\
\hline Death & $8(4.9)$ & $16(4.9)$ \\
\hline Any serious $A E$ & $52(32.1)$ & $88(27.2)$ \\
\hline Treatment discontinuation due to $\mathrm{AE}$ & $18(11.1)$ & $24(7.4)$ \\
\hline Any $A E$ & $151(93.2)$ & 301 (92.9) \\
\hline Mild $^{\mathrm{b}}$ & $44(27.2)$ & $87(26.9)$ \\
\hline Moderate $^{b}$ & $68(42.0)$ & $150(46.3)$ \\
\hline Severe $^{b}$ & $30(18.5)$ & $53(16.4)$ \\
\hline Very severe ${ }^{b}$ & $9(5.6)$ & $11(3.4)$ \\
\hline \multicolumn{3}{|l|}{ Frequent $\mathrm{AEs}$ ( $\geq 10 \%$ in either arm) } \\
\hline Fall & $87(53.7)$ & $192(59.3)$ \\
\hline Urinary tract infection & $32(19.8)$ & $57(17.6)$ \\
\hline Skin laceration & $18(11.1)$ & 43 (13.3) \\
\hline Contusion & $24(14.8)$ & $42(13.0)$ \\
\hline Constipation & $15(9.3)$ & $36(11.1)$ \\
\hline Headache & $22(13.6)$ & $31(9.6)$ \\
\hline
\end{tabular}

Data are number (\%) of participants having at least one event. AEs are listed by preferred

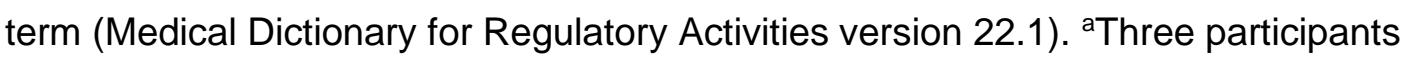
randomized to placebo received one dose of gosuranemab and are included in the gosuranemab group for safety analyses. ${ }^{b}$ Each participant counted once at maximum severity. 
Table 4. AEs occurring in $\geq 5 \%$ of participants and serious AEs occurring in $\geq 2 \%$ of participants, in either group

\begin{tabular}{|c|c|c|}
\hline & $\begin{array}{c}\text { Placebo } \\
n=162\end{array}$ & $\begin{array}{c}\text { Gosuranemab } \\
n=324^{\mathrm{a}}\end{array}$ \\
\hline \multicolumn{3}{|l|}{$A E s$ in $\geq 5 \%$ in either group } \\
\hline Fall & 87 (53.7) & $192(59.3)$ \\
\hline Urinary tract infection & $32(19.8)$ & $57(17.6)$ \\
\hline Skin laceration & $18(11.1)$ & $43(13.3)$ \\
\hline Contusion & $24(14.8)$ & $42(13.0)$ \\
\hline Constipation & $15(9.3)$ & $36(11.1)$ \\
\hline Headache & $22(13.6)$ & $31(9.6)$ \\
\hline Nasopharyngitis & $14(8.6)$ & $28(8.6)$ \\
\hline Skin abrasion & $10(6.2)$ & $28(8.6)$ \\
\hline Hematoma & $12(7.4)$ & $24(7.4)$ \\
\hline Diarrhea & $9(5.6)$ & $23(7.1)$ \\
\hline Head injury & $8(4.9)$ & $21(6.5)$ \\
\hline Arthralgia & $7(4.3)$ & $19(5.9)$ \\
\hline Musculoskeletal pain & $6(3.7)$ & $19(5.9)$ \\
\hline Dizziness & $13(8.0)$ & $18(5.6)$ \\
\hline Rib fracture & $9(5.6)$ & $18(5.6)$ \\
\hline Dysphagia & $12(7.4)$ & $17(5.2)$ \\
\hline Pain in extremity & $7(4.3)$ & $17(5.2)$ \\
\hline Pneumonia & $5(3.1)$ & $17(5.2)$ \\
\hline Insomnia & $14(8.6)$ & $16(4.9)$ \\
\hline Back pain & $9(5.6)$ & $15(4.6)$ \\
\hline \multicolumn{3}{|c|}{ Serious $A E s \geq 2 \%$ in either group } \\
\hline Fall & $10(6.2)$ & $41(12.7)$ \\
\hline Pneumonia & 0 & $10(3.1)$ \\
\hline Aspiration pneumonia & $8(4.9)$ & $6(1.9)$ \\
\hline Dysphagia & $5(3.1)$ & $3(0.9)$ \\
\hline
\end{tabular}

Data are number (\%) of participants having at least one event. AEs are listed by preferred term (Medical Dictionary for Regulatory Activities, version 22.1.)

aThree participants randomized to placebo received one dose of gosuranemab and are included in the gosuranemab group for safety analyses 


\begin{tabular}{lcc}
\hline & $\begin{array}{c}\text { Placebo } \\
n=162\end{array}$ & $\begin{array}{c}\text { Gosuranemab } \\
n=324^{\mathrm{a}}\end{array}$ \\
\hline Pneumonia $^{\mathrm{b}}$ & $5(3.1)$ & $17(5.2)$ \\
Aspiration pneumonia $^{\mathrm{b}}$ & $8(4.9)$ & $6(1.9)$ \\
$\begin{array}{l}\text { Any pneumonia or aspiration } \\
\text { pneumonia }\end{array}$ & $13(8.0)$ & $21(6.5)$ \\
$\begin{array}{l}\text { Any event of infectious } \\
\text { pneumonia/lower respiratory infection } \\
\text { or aspiration }\end{array}$ & $25(15.4)$ & $41(12.7)$
\end{tabular}

Data are number (\%) of participants having at least one event.

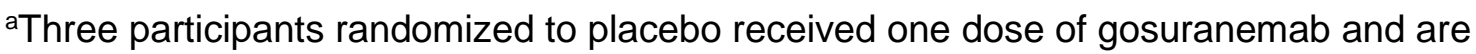
included in the gosuranemab group for safety analyses.

'These analyses include only the preferred terms of "pneumonia" and "pneumonia aspiration," as indicated.

'This analysis is based on the Infective Pneumonia Standardized Medical Dictionary for Regulatory Activities Query (narrow and broad preferred terms) plus preferred terms related to aspiration ("pneumonia aspiration," "aspiration," and "foreign body aspiration"). 


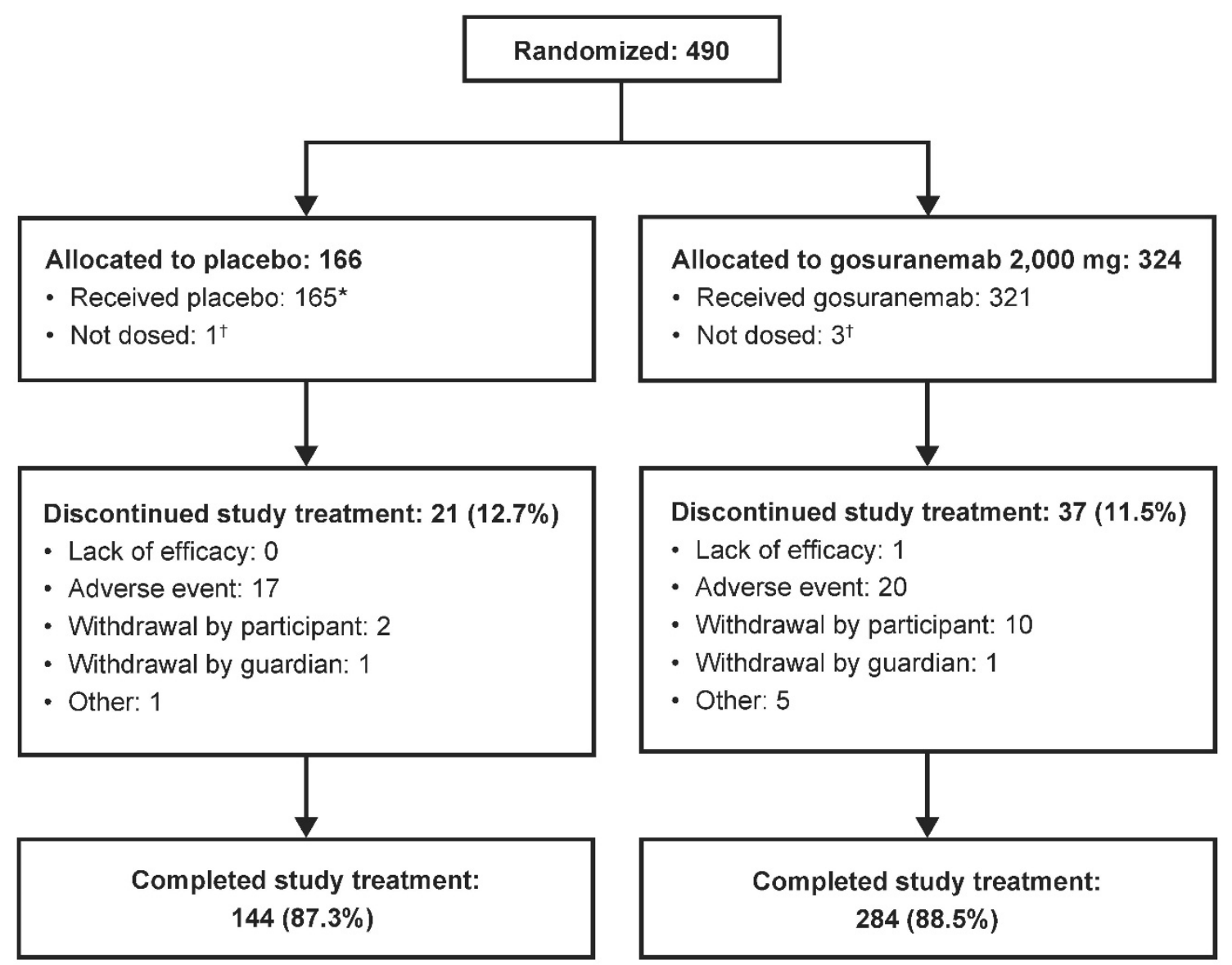

Extended Data Fig. 1 | Participant disposition in the PASSPORT study. Percentages are based on the number of participants who received study drug. *Three participants randomly assigned to placebo received one dose of gosuranemab. ${ }^{\dagger}$ One participant assigned to placebo was not dosed due to abnormal vital signs, and three participants assigned to gosuranemab were not dosed because they failed to meet randomization criteria. 


\section{Methods}

\section{Study design and participants}

In this randomized, double-blind, placebo-controlled, parallel-group phase 2 study, we evaluated the efficacy and safety of gosuranemab in adults with a diagnosis of probable or possible PSP and symptoms for 5 years or less at baseline. Diagnosis of PSP was based on a history of postural instability or fall during the first 3 years of symptom presence (consistent with the recent MDS PSP diagnostic criteria), ${ }^{2,55}$ the presence of vertical supranuclear gaze palsy or slow velocity of vertical saccades, and an akinetic-rigid syndrome. ${ }^{5}$ At screening, the main eligibility criteria were $41-86$ years of age, body weight of 43-120 kg, ability to ambulate independently or with limited assistance, a score of at least 20 on the Mini-Mental State Examination score, living outside a nursing home or dementia care facility, and no other significant neurological or psychiatric disorders including Alzheimer's disease, dementia with Lewy bodies, prion disease, Parkinson's disease, hydrocephalus or clinically relevant cerebrovascular disease. Participants could not have been treated within 4 weeks of screening or initiate treatment during the study with memantine, acetylcholinesterase inhibitors, antipsychotic agents or mood stabilizers. Dosing of antiparkinsonian drugs had to be stable for 60 days before enrollment and other chronic medications were to be stable for 30 days; all had to be held unchanged for the duration of the trial unless patient safety mandated a change. Participants were recruited at 90 outpatient specialized movement disorders clinic study sites across 13 countries. Study visits (including screening visits) occurred between April 24, 2017 and September 6, 2019.

The study protocol was approved by Advarra's institutional review board (https://www.advarra.com/irb-services/sponsors-cros/) for 6 US sites: Rutgers Robert Wood Johnson Medical School, New Brunswick, NJ; University of Florida Center For Movement Disorders and Neurorestoration, Gainesville, FL; Banner Sun Health Research Institute, Sun City, AZ; University of South Florida - Morsani College of Medicine, Tampa, FL; QUEST Research Institute, Farmington Hills, MI; and St. Joseph's Hospital \& Medical Center/Barrow 
Neurology Clinics, Phoenix, AZ. For all other sites, the institutional review board or ethics committee at the institutions listed for the PASSPORT study group investigators approved the study protocol. Enrolled study participants and their caregivers provided written informed consent. The study was conducted in accordance with Good Clinical Practice principles. An independent Data Monitoring Committee composed of four independent clinicians and one independent statistician with neurological clinical trial experience was established to review unblinded safety data and make recommendations regarding continuation, modification or termination of the study for safety concerns.

\section{Randomization and blinding}

Enrolled participants were randomized in a 2:1 ratio to receive gosuranemab 2,000 mg or placebo (dextrose 5\%) administered intravenously every 4 weeks for approximately 48 weeks (up to 13 times). Randomization was performed centrally using interactive response technology and stratified by country and Color Trails ${ }^{56}$ test part 2 score at screening ( $\leq 170$ seconds versus $>170$ seconds). All participants and study personnel were blinded to treatment assignment, with the exception of the pharmacists who prepared the study drugs and provided them to blinded personnel. At the end of the placebo-controlled period, participants could opt to continue into an open-label long-term extension period in which all participants received gosuranemab 2,000 mg every 4 weeks. No study drug dose escalations or reductions were permitted.

Gosuranemab was provided by the study sponsor as a sterile solution for parenteral administration at a strength of $50 \mathrm{mg} / \mathrm{ml}$. Each vial contained an extractable dose of 1000 $\mathrm{mg}$ in $20 \mathrm{ml}$ or an extractable dose of $2000 \mathrm{mg}$ in $40 \mathrm{ml}$. Gosuranemab infusions were administered undiluted or after dilution in $0 / 9 \%$ saline or $5 \%$ dextrose for injection.

\section{Procedures}

Study visits occurred approximately every 4 weeks, at which times study drug was administered. Safety was monitored throughout the study. Urine and blood samples were 
collected for laboratory tests at weeks $0,4,8,12,24,36,48$ and 52; blood samples for pharmacokinetic analyses were also collected at weeks 0, 4, 24 and 48 (predose and end of infusion) and at weeks 12, 36 and 52 (predose only). CSF samples were collected at selected sites during screening and at week 52. Efficacy assessments were performed at weeks $0,12,24,36,48$ and 52 . Structural MRI using a magnetization-prepared 3D T1weighted sequence was performed during screening and at weeks 24 and 52 . Volumetric quantification of PSP-relevant brain structures, specifically the lateral ventricles, whole brain, midbrain and pons, were performed using an atlas-based segmentation technique. ${ }^{57}$ Participants who discontinued study treatment early were encouraged to return to the clinic at week 52 to complete the scheduled assessments and received a follow-up call 30 days after their last dose for safety assessment.

\section{Outcomes}

The primary efficacy outcome was change from baseline in the PSPRS score at week 52 in participants treated with gosuranemab relative to participants treated with placebo. The PSPRS is a validated instrument that is sensitive to disease progression ${ }^{6}$ and comprises 28 items in the subscales of daily activity (history), mentation, bulbar, ocular motor, limb motor and gait/midline (score range: 0 [normal] to 100). The primary safety outcomes were frequency of deaths, serious AEs, AEs leading to discontinuation and grade 3 and 4 laboratory abnormalities graded by numerical criteria from the Common Terminology Criteria for Adverse Events version 4.0.3.

Key secondary efficacy endpoints included change from baseline at week 52 on the MDS-UPDRS Part II (Motor Experiences of Daily Living), ${ }^{30}$ CGI-C ${ }^{58}$ score at Week 52 and change from baseline at week 52 on the PSP cognitive composite battery and PSP-QoL. ${ }^{29}$ The MDS-UPDRS Part II is a validated 13-item questionnaire that assesses the motor aspects of experiences of daily living. ${ }^{30}$ The CGI-C measures change in a participant's clinical status from a specific point in time on a 7-point scale, ranging from 1 (very much improved) to 7 (very much worse). ${ }^{58}$ The PSP cognitive composite battery identifies and 
characterizes abnormal cognitive decline in participants with PSP within the domains of memory and learning, visual-motor function and working memory and executive. It is composed of 11 subtests of the Repeatable Battery for the Assessment of Neuropsychological Disease Severity (RBANS ${ }^{59}$; picture naming is excluded) plus letternumber sequencing and phonemic fluency tests (J. Jaeger, T.D., L.Y., J.O., Y.L., S.B.H., C.J. Castrillo-Viguerra, manuscript in preparation). The PSP-QoL, a validated patientreported outcome measure developed specifically for people with PSP, consists of three subscales, including a 22-item cognitive subscale and a 23-item physical subscale that assess the physical and mental impact of the disorder, and a visual analog scale (VAS) that assesses the patient's satisfaction with life overall. Items are given a 5-response option format (range, 0 [no problem] to 4 [extreme problem]) and the subscale sum scores are converted to a $0-100$ scale. $^{29}$

Other secondary endpoints included change from baseline at week 48 in the SEADL scale, an 11-point, single-item measure of overall functional independence (range: 100\% [complete independence] to $0 \%$ [complete dependence; bedridden] ${ }^{60}$; change from baseline at week 52 in CGI-S, which measures PSP symptomatology on a 7-point scale ranging from normal to extremely ill ${ }^{18}$; and change at week 48 in a phonemic fluency test, ${ }^{61}$ in which participants have 1 minute to give as many words as possible that start with a selected letter. Absolute change from baseline in volumes of the lateral ventricles, whole brain, midbrain and pons were assessed at week 52 on MRI scans (secondary endpoint). The exploratory endpoints of gosuranemab concentrations in blood and CSF were quantified with a chemiluminescent immunoassay at QPS (Newark, DE, USA) and unbound N-terminal tau CSF concentrations were quantified using validated fit-for-purpose immunoassays (Meso Scale Diagnostics, Gaithersburg, MD, USA) as previously described. ${ }^{18}$

\section{Statistical analyses}

Anticipating a dropout rate of approximately $25 \%$, the sample size of 459 participants was determined to provide $80 \%$ power to detect a difference of 3.2 points in the change in 
PSPRS total score from baseline to week 52 for gosuranemab relative to placebo, using a two-sided two-sample $t$-test with an alpha level set at 0.05 assuming a standard deviation of 9.95. ${ }^{62}$ Efficacy analyses were performed in the intention-to-treat population, which consisted of all randomized participants who received at least one dose of blinded study medication, and were conducted according to assigned treatment groups. A prespecified hierarchical testing procedure in the following order was used to test key secondary endpoints for controlling the type 1 error rate: MDS-UPDRS Part II, CGI-C, PSP cognitive composite battery, and PSP-QoL. If statistical significance was not achieved for a key secondary endpoint, all key secondary endpoint(s) of a lower rank were not considered statistically significant. The efficacy endpoints were analyzed using a mixed model repeated measures model with fixed effects of treatment group, time (categorical), treatment group by time interaction, baseline value, baseline value by time interaction, region (US or non-US) and baseline Color Trails 2 test ( $\leq 170$ or $>170$ seconds). Missing data were assumed to be missing at random. Additional exploratory analyses based on the primary mixed model repeated measures model were conducted by adding Color Trails 2 test by visit interaction; and by adding Color Trails 2 test by visit interaction, Color Trails 2 test by treatment interaction, and Color Trails 2 test by visit by treatment group interaction. The efficacy MRI population, which consisted of participants in the intention-to-treat population who had at least one measurable brain volumetric measurement, was used for the volumetric MRI analysis. There was no normalization of MRI volumes by intracranial volume. The same mixed model repeated measures model used for the efficacy endpoints was applied for the MRI analysis. Percentage change in CSF N-terminal tau and neurofilament light chain was analyzed in the CSF pharmacodynamic population (subset of the safety population with at least one sample evaluable for exploratory CSF biomarkers) using an analysis of covariance model fitted with change/percentage change from baseline, adjusting for treatment group, baseline CSF value, baseline Color Trails 2 test ( $\leq 170$ or $>170$ seconds) and region. Serum and CSF gosuranemab concentrations were respectively analyzed in the serum and CSF pharmacokinetics analysis populations, which were the subsets of the safety population that 
had at least one measurable postbaseline gosuranemab concentration in serum and CSF, respectively. Safety analyses were performed using safety treatment groups for treated participants (i.e., enrolled participants who received at least one dose of study treatment). If a participant received at least one dose of gosuranemab then they were included in the gosuranemab safety treatment group. If a participant only received doses of placebo, they were included in the placebo safety treatment group. The protocol described a possible interim analysis for efficacy before the last enrolled participant completed the week 52 visit; however, no interim analyses were done for the placebo-controlled period of the study. All analyses were done with SAS, version 9.4.

\section{Data availability}

To request access to data, please visit http://www.biogenclinicaldatarequest.com. The individual participant data collected during the trial, which supports the research proposal, will be available to qualified scientific researchers after anonymization and upon approval of the research proposal. Anonymisation of the datasets is necessary to allow data to be shared ethically and legally, and to maximize their significant social, environmental, and economic value, whilst preserving confidentiality of the individuals who participated in studies conducted by Biogen. An initial status update of the request will take approximately two (2) business days after the request's submission date. The requester will be notified of the outcome of the request in approximately thirty (30) business days. 


\section{Supplementary materials}

Supplement to: Tien Dam, M.D., Adam L. Boxer, M.D., Lawrence I. Golbe, M.D., et al. Safety and efficacy of anti-tau monoclonal antibody gosuranemab in progressive supranuclear palsy: a phase 2, randomized, placebo-controlled trial

\section{PASSPORT Investigators}

Ikuko Aiba ${ }^{11}$, Angelo Antonini ${ }^{13}$, Diana Apetauerova ${ }^{14}$, Jean-Philippe Azulay ${ }^{15}$, Ernest Balaguer Martinez $^{16}$, Jee Bang ${ }^{17}$, Paolo Barone ${ }^{18}$, Matthew Barrett ${ }^{19}$, Danny Bega ${ }^{20}$, Daniela Berg ${ }^{21}$, Koldo Berganzo Corrales ${ }^{22}$, Yvette Bordelon ${ }^{23}$, Adam L Boxer ${ }^{2}$, Moritz Brandt ${ }^{24}$, Norbert Brueggemann ${ }^{25}$, Giovanni Castelnovo ${ }^{26}$, Roberto Ceravolo ${ }^{27}$, Rosalind Chuang ${ }^{28}$, Sun Ju Chung $^{29}$, Alistair Church ${ }^{30}$, Jean-Christophe Corvol ${ }^{10}$, Paola Cudia ${ }^{13}$, Marian Dale ${ }^{31}$, Luc Defebvre $^{32}$, Sophie Drapier ${ }^{33}$, Erika D Driver-Dunckley ${ }^{34}$, Georg Ebersbach ${ }^{35}$, Karla M Eggert ${ }^{36}$, Aaron Ellenbogen ${ }^{37}$, Alexandre Eusebio ${ }^{15}$, Andrew H Evans ${ }^{38}$, Natalia Fedorova ${ }^{39}$, Elizabeth Finger ${ }^{40}$, Alexandra Foubert-Samier ${ }^{41}$, Boyd Ghosh ${ }^{42}$, Lawrence Golbe ${ }^{3}$, Francisco Grandas Perez $^{43}$, Murray Grossman ${ }^{44}$, Deborah Hall ${ }^{45}$, Kyoko Hamada ${ }^{46}$, Kazuko Hasegawa ${ }^{47}$, Guenter Hoeglinger $^{4}$, Lawrence Honig ${ }^{48}$, David Houghton ${ }^{49}$, Xuemei Huang ${ }^{50}$, Stuart Isaacson ${ }^{51}$, SeongBeom Koh ${ }^{52}$, Jaime Kulisevsky Bojarski ${ }^{53}$, Anthony E. Lang ${ }^{9}$, Peter Nigel Leigh ${ }^{54}$, Irene Litvan ${ }^{8}$, Juan Jose Lopez Lozano ${ }^{55}$, Jose Luis Lopez-Sendon Moreno ${ }^{56}$, Albert Christian Ludolph ${ }^{57}$, Ma Rosario Luquin Piudo ${ }^{58}$, Irene Martinez Torres ${ }^{59}$, Nikolaus McFarland ${ }^{60}$, Wassilios Meissner ${ }^{41}$, Tiago Mestre ${ }^{61}$, Pablo Mir Rivera ${ }^{62}$, Eric Molho ${ }^{63}$, Britt Mollenhauer ${ }^{64}$, Huw R Morris ${ }^{7}$, Miho Murata $^{65}$, Tomokazu Obi6 ${ }^{66}$ Fabienne Ory Magne ${ }^{67}$, Padraig O'Suilleabhain ${ }^{68}$, Rajesh Pahwa ${ }^{69}$, Alexander Pantelyat ${ }^{17}$, Nicola Pavese ${ }^{70}$, Dmitry Pokhabov ${ }^{71}$, Johannes Prudlo ${ }^{72}$, Federico Rodriguez-Porcel ${ }^{31}$, James Rowe ${ }^{73}$, Joseph Savitt ${ }^{74}$, Alfons Schnitzler ${ }^{75}$, Joerg B Schulz ${ }^{76}$, Klaus Seppi $^{77}$, Binit Shah ${ }^{19}$, Holly Shill ${ }^{78}$, David Shprecher ${ }^{79}$, Maria Stamelou ${ }^{80}$, Malcolm Steiger ${ }^{81}$, Yuji Takahashi $^{65}$, Hiroshi Takigawa ${ }^{82}$, Carmela Tartaglia ${ }^{83}$, Lars Toenges ${ }^{84}$, Daniel Truong ${ }^{85}$, Winona Tse $^{86}$, Paul Tuite ${ }^{87}$, Dieter Volc ${ }^{88}$, Anne-Marie A Wills ${ }^{89}$, Dirk Woitalla ${ }^{90}$, Tao Xie ${ }^{91}$, Tatsuhiko Yuasa $^{92}$, Sarah Elizabeth Zauber ${ }^{93}$, Theresa Zesiewicz ${ }^{94}$

${ }^{13}$ San Camillo Hospital IRCCS, Venice Lido, Italy; ${ }^{14}$ Lahey Hospital and Medical Center, Burlington, MA, USA; ${ }^{15}$ Assistance Publique Hapitaux De Marseille, Marseille, France, ${ }^{16}$ Hospital General de Catalunya, Barcelona, Spain, ${ }^{17}$ The Johns Hopkins University, Baltimore, MD, USA; ${ }^{18} \mathrm{AOU}$ San Giovanni di Dio e Ruggi d'Aragona University di Salerno, Salerno, Italy; 
${ }^{19}$ University of Virginia Health System, Charlottesville, VA, USA; ${ }^{20}$ Northwestern University, Chicago, IL, USA; ${ }^{21}$ UKSH - Campus Kiel, Kiel, Germany; ${ }^{22}$ Hospital De Cruces, Barakaldo, Spain; ${ }^{23}$ University of California, Los Angeles, CA, USA; ${ }^{4}$ Universitatsklinikum Carl Gustav Carus Dresden, Dresden, Germany, ${ }^{25}$ University Hospital Schleswig-Holstein, Luebeck, Germany; ${ }^{26}$ Centre Hospitalier Universitaire de Nimes - Hopital Universitaire Caremeau, Nimes, France; ${ }^{27}$ University Hospital of Pisa, Pisa, Italy; ${ }^{28}$ Swedish Health Services, Seattle, WA, USA; ${ }^{29}$ Asan Medical Center, Seoul, Republic of Korea; ${ }^{30}$ Aneurin Bevan University Health BoardClinical Research and Innovation Centre - St Woolos Hospital, Newport, UK; ${ }^{31}$ Medical University of South Carolina, Charleston, SC, USA; ${ }^{32}$ Centre Hospitalier Regional Universitaire ) de Lille - Hopital Roger Salengro, Lille, France; ${ }^{33} \mathrm{CHU}$ de Rennes - Hopital de Pontchaillou, Rennes, France; ${ }^{34}$ Mayo Clinic Arizona - Scottsdale, Scottsdale, AZ, USA; ${ }^{35}$ Movement Disorders Clinic, Beelitz-Heilstatten, Germany; ${ }^{36}$ Philipps Universitat Marburg, Marburg, Germany; ${ }^{37}$ QUEST Research Institute, Farmington Hills, MI, USA; ${ }^{38}$ The Royal Melbourne Hospital (RMH)-Flemington Neurology - North Melbourne, North Melbourne, Australia; ${ }^{39}$ Russian Medical Academy of Postgraduate Education, Moscow, Russia; ${ }^{40}$ Parkwood Institute, London, Ontario, Canada; ${ }^{41} \mathrm{CHU}$ De Bordeaux Parkinson Expert Centre, IMNC Hopital Pellegrin, Bordeaux, France; ${ }^{42}$ University Hospital Southampton NHS Foundation Trust, Southampton UK; ${ }^{43}$ Hospital General Universitario Gregorio Maranon, Madrid, Spain; ${ }^{44}$ Hospital of the University of Pennsylvania, Philadelphia, PA, USA; ${ }^{45}$ Rush University Medical Centre Chicago, IL USA; ${ }^{46}$ Shinsapporo Neurosurgical Hospital, Sapporo, Japan; ${ }^{47}$ National Hospital Organization Sagamihara National Hospital, Sagamihara, Japan; ${ }^{48}$ Columbia University College of Physicians and Surgeons - Gertrude H. Sergievsky Center, New York, NY, USA; ${ }^{9}$ Ochsner Medical Center, New Orleans, LA, USA; ${ }^{50}$ Penn State University-Milton S. Hershey Medical Center, Hershey, PA USA; ${ }^{11}$ Parkinson's Disease And Movement Disorder Center Of Boca Raton, Boca Raton, FL, USA; ${ }^{52}$ Korea University Guro Hospital, Seoul, Republic of Korea; ${ }^{53}$ Hospital de la Santa Creu i Sant Pau, Barcelona, Spain; ${ }^{54}$ Brighton and Sussex Medical School Trafford Centre for Biomedical Research, Brighton, UK; ${ }^{55}$ Clinica Ruber Internacional, Madrid, Spain; ${ }^{56}$ Hospital Universitario Ramon y Cajal, Madrid, Spain; ${ }^{57}$ Universitats- und Rehabilitationskliniken UIm,Ulm, Germany; ${ }^{58}$ Clinica Universidad De Navarra, Pamplona, Spain; ${ }^{59}$ Hospital la FE, Valencia, Spain; ${ }^{60}$ University of Florida Center For Movement Disorders and Neurorestoration, Gainesville, FL, USA; ${ }^{61}$ The Ottawa Hospital - Civic Campus, University Of Ottawa, Ottawa, Canada; ${ }^{62}$ Hospital Universitario Virgen del Rocio, Sevilla, Spain; ${ }^{63}$ Albany Medical College, Albany, NY, USA; ${ }^{4}$ Paracelsus-Elena-Klinik Kassel, Kassel, Germany; ${ }^{65}$ Musashi Hospital, Kodaira-Shi, Japan; ${ }^{66}$ National Hospital Organization - Shizuoka Institute of 
Epilepsy and Neurological Disorders, Shizuoka, Japan; ${ }^{67}$ Hopital Purpan - Batiment Pierre Paul Riquet, Toulouse, France; ${ }^{68}$ University of Texas Southwestern Medical Center - Clinical Center For Movement Disorders, Dallas, TX, USA; ${ }^{69}$ The University of Kansas Medical Center Parkinson's Disease and Movement Disorder Center, Kansas City, KS, USA; ${ }^{70}$ The Newcastle upon Tyne Hospitals NHS Foundation Trust - Campus for Ageing and Vitality, Newcastle upon Tyne, UK; ${ }^{71}$ Federal State Budgetary Institution, Federal Siberian Scientific Clinical Center of Federal Medical-Biological Agency, Krasnoyarsk, Russia; ${ }^{72}$ Universitaet Rostock Universitaetsmedizin Rostock, Rostock, Germany; ${ }^{73}$ Cambridge University, Cambridge, UK; ${ }^{74}$ University of Maryland School of Medicine, Baltimore, MD, USA; ${ }^{75}$ Center for Movement Disorders and Neuromodulation-University Hospital Dusseldorf, Dusseldorf, Germany; ${ }^{76}$ Uniklinik RWTH Aachen Medizinische Klinik III, Aachen, Germany; ${ }^{77}$ Medizinische Universitaet Innsbruck, Innsbruck, Austria; ${ }^{78} \mathrm{St}$. Joseph's Hospital and Medical Center/Barrow Neurology Clinics, Phoenix, AZ, USA; ${ }^{79}$ Banner Sun Health Research Institute, Sun City, AZ, USA; ${ }^{80}$ Hygeia Hospital, Marousi, Greece; ${ }^{81}$ The Walton Center - NHS Foundation Trust, Liverpool, UK; ${ }^{82}$ Tottori University Hospital, Yonago, Japan; ${ }^{83}$ Toronto Western Hospital, University Health Network Movement Disorders Centre, Toronto, Canada; ${ }^{84} \mathrm{St}$. Josef - Hospital Bochum, Kardiologische Studienambulanz, Bochum, Germany; ${ }^{85}$ The Parkinson's and Movement Disorder Institute, Fountain Valley, CA, USA; ${ }^{86}$ Mount Sinai Movement Disorders Center, New York,NY, USA; ${ }^{87}$ University of Minnesota Medical Center - Fairview - Neurology Clinic, Minneapolis, MN, USA; ${ }^{88}$ Prosenex Ambulatoriumsbetriebs $\mathrm{GmbH}$ - Studienzentrum, Vienna, Austria; ${ }^{89}$ Massachusetts General Hospital Cancer Center, Boston, MA, USA; ${ }^{90}$ St. JosefKrankenhaus, Essen-Kupferdreh, Essen, Germany; ${ }^{91}$ The University of Chicago Medicine Center for Parkinson's Disease and Movement Disorders, Chicago, IL, USA; ${ }^{92}$ Kamagaya General Hospital, Kamagaya-City, Japan; ${ }^{93}$ Indiana University Health Physicians - Neurology Indianapolis, Indianapolis, IN, USA; ${ }^{94}$ University of South Florida - Morsani College of Medicine, Tampa, FL, USA 
Supplementary Table S1. Results for change from baseline in total PSPRS score at Week 52 for various exploratory analyses (intention-to-treat population)

\begin{tabular}{|l|c|c|c|c|}
\hline & $\begin{array}{c}\text { Adjusted } \\
\text { change from } \\
\text { baseline at } \\
\text { Week 52 in } \\
\text { Placebo }\end{array}$ & $\begin{array}{c}\text { Adjusted } \\
\text { change from } \\
\text { baseline at } \\
\text { Week 52 in } \\
\text { gosuranemab }\end{array}$ & $\begin{array}{c}\text { Treatment } \\
\text { difference vs } \\
\text { placebo (SE) }\end{array}$ & P value* \\
\hline $\begin{array}{l}\text { Primary analysis MMRM } \\
\text { model }\end{array}$ & 10.59 & 10.41 & $-0.18(0.920)$ & 0.8483 \\
\hline $\begin{array}{l}\text { Color Trails 2 test by } \\
\text { visit interaction added }\end{array}$ & 10.01 & 9.67 & $-0.34(0.910)$ & 0.7082 \\
\hline $\begin{array}{l}\text { Color Trails 2 test by } \\
\text { visit interaction, Color } \\
\text { Trails 2 test by } \\
\text { treatment interaction, } \\
\text { and Color Trails 2 test } \\
\text { by visit by treatment } \\
\text { interaction added }\end{array}$ & 10.02 & 9.67 & $-0.36(0.992)$ & 0.7202 \\
\hline
\end{tabular}

$P$ values are nominal. All $P$ values were tested two-sided. $F$ statistics were used for the statistical testing with the Kenward-Rodgers method used for denominator degrees of freedom. 
Supplementary Table S2. Summary of participants by treatment group and by country (intention-to-treat population)

\begin{tabular}{lcc}
\hline \multicolumn{1}{c}{ Country } & Placebo & Gosuranemab \\
Australia & $\boldsymbol{n}=\mathbf{1 6 5}$ & $\boldsymbol{n}=\mathbf{3 2 1}$ \\
Austria & 1 & 0 \\
Canada & 1 & 12 \\
Germany & 6 & 10 \\
Spain & 28 & 52 \\
France & 24 & 40 \\
United Kingdom & 18 & 46 \\
Greece & 9 & 15 \\
Italy & 3 & 4 \\
Japan & 9 & 16 \\
South Korea & 16 & 23 \\
Russia & 2 & 6 \\
United States & 5 & 11 \\
\hline
\end{tabular}


Supplementary Figure S1. Scatterplots of (a) CSF unbound N-terminal tau (pg/ml) and (b) CSF neurofilament light chain $(\mathrm{pg} / \mathrm{ml})$ for individual participants. Note: only participants who had both baseline and Week 52 data are included.
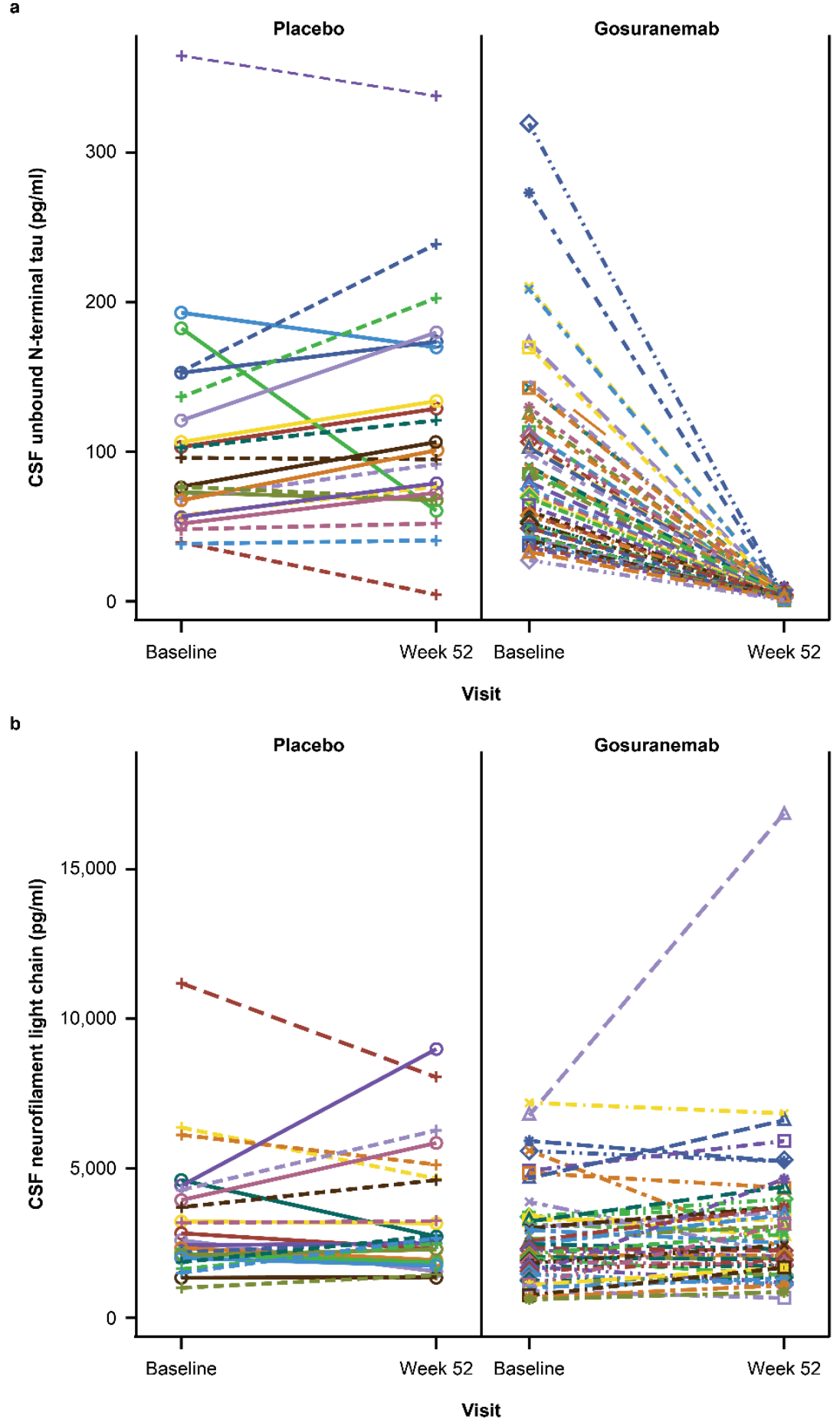